\title{
Justification of the Zakharov model from Klein-Gordon-wave systems
}

\author{
Thierry Colin ${ }^{1}$, Géraldine Ebrard ${ }^{1,2}$, Gérard Gallice ${ }^{2}$ and Benjamin Texier ${ }^{1}$ \\ ${ }^{1}$ Mathématiques Appliquées de Bordeaux, Université Bordeaux 1 et CNRS \\ UMR 5466, 351 cours de la libération, 33405 Talence cedex, France \\ ${ }^{2}$ DEV/SIS, CEA CESTA, BP 2 - 33114 Le Barp, France.
}

\begin{abstract}
We study semilinear and quasilinear systems of the type KleinGordon-waves in the high-frequency limit. These systems are derived from the Euler-Maxwell system describing laser-plasma interactions. We prove the existence and the stability of high-amplitude WKB solutions for these systems. The leading terms of the solutions satisfy Zakharov-type equations. The key is the existence of transparency equalities for the Klein-Gordon-waves systems. These equalities are comparable to the transparency equalities exhibited by J.-L. Joly, G. Métivier and J. Rauch for Maxwell-Bloch systems.
\end{abstract}

Keywords. Laser-plasma interactions, Klein-Gordon-waves systems, Zakharov equation, high-amplitude WKB solutions, transparency.

AMS Classification. 35L60, 35Q55, 78M35. 


\section{Introduction}

\subsection{Physical context and motivations}

The construction of powerful lasers enables the study of highly nonlinear regime in plasma physics. Therefore, nonlinear models have to be used. The simplest and most popular model for nonlinear interaction of a laser and a plasma is the so-called Zakharov system [28]. It is used for dense, hot plasmas. A typical version of the scalar Zakharov system is

$$
\left\{\begin{array}{c}
i \partial_{t} u+\Delta u=n u \\
\partial_{t}^{2} n-\Delta n=\Delta|u|^{2}
\end{array}\right.
$$

This system describes the nonlinear interaction of the envelope of the electric field $u$ with the low-frequency ionic fluctuation of density in the plasma $n$. The local in time Cauchy problem for (1.1) has been well understood since the work of T. Ozawa and Y. Tsutsumi [20] for regular solutions and J. Ginibre, Y. Tsutsumi and G. Velo [11] (based on the ideas of J. Bourgain) for weak solutions (see also the work of S. Schochet and M. Weinstein for an alternate proof [24]). A vectorial version of the Zakharov equation

$$
\left\{\begin{array}{ccc}
i \partial_{t}(\nabla \psi)+\Delta(\nabla \psi) & = & \nabla \Delta^{-1} \nabla \cdot(n \nabla \psi), \\
\partial_{t}^{2} n-\Delta n & = & \Delta|\nabla \psi|^{2},
\end{array}\right.
$$

has been studied by B. Bidegaray [1]. This version is closer to the physics. L. Glangetas and F. Merle contructed blow-up solutions in two dimensions for $(1.1)[12,13]$. The numerical study of $(1.1)$ and (1.2) is also quite complete. Order 2, energy preserving schemes were introduced by R. Glassey [14, 15]. Physicists usually prefer spectral schemes (see [21] and [22]) coupling several systems of the form (1.1).

In contrast, the justification of systems (1.1) or (1.2) from the bifluid 
Euler-Maxwell system is far from satisfactory (see the book of C. Sulem and P.-L. Sulem [25], chapter 13). In this paper, we will consider only regular solutions of (1.1) obtained from example by T. Ozawa and Y. Tsutsumi's method and we will address the following question: in what sense solutions of (1.1) describe accurately the solutions of the Euler-Maxwell system ? Of course we do not have any definitive answer (see Remark 1.10 about the extension of our result to the full Euler-Maxwell equations). For the setting of a quite complete Euler-Maxwell model see [27] or [3] for a formal derivation of systems used in [22]. A first justification of asymptotic expansions for small solutions of the Euler-Maxwell equations leading to the Zakharov equations is given in $[27]$.

The aim of this paper is to give some justifications of such expansions for larger solutions starting from simplified systems of the form Klein-Gordonwave. Unfortunately, our proof does not apply to the Euler-Maxwell system. The Klein-Gordon-waves systems for which our proof is valid are intermediate between Euler-Maxwell and Zakharov. We will construct such systems and explain why we can justify such asymptotics for these systems. We also introduce a semilinearization process that enables us to tranform simplified quasilinear systems used to describe laser-plasma interactions into semilinear systems to which our theory applies.

A global existence result for the Klein-Gordon-wave system (1.10) (for fixed $\varepsilon$ ) is given by T. Ozawa, K. Tsutaya and Y. Tsutsumi in [19].

Our method relies on the work of J.-L. Joly, G. Métivier and J. Rauch in the nineties. In the next section, we introduce the mathematical context and explain to what extent this work is related to their work. 


\subsection{Mathematical context}

Consider hyperbolic semilinear systems of the form

$$
\mathcal{L}^{\varepsilon}\left(\partial_{t}, \partial_{x}\right) U^{\varepsilon}=\left(\partial_{t}+\mathcal{A}\left(\partial_{x}\right)+\frac{1}{\varepsilon} \mathcal{L}_{0}\right) U^{\varepsilon}=F\left(U^{\varepsilon}\right),
$$

with the initial datum

$$
U^{\varepsilon}(t=0, x)=\varepsilon^{p} A(x) e^{i k x / \varepsilon}+c . c .
$$

where $\mathcal{A}\left(\partial_{x}\right)=\sum_{j} \mathcal{A}_{j} \partial_{j}$; the matrices $\mathcal{A}_{j}$ are symmetric and $\mathcal{L}_{0}$ is skewsymmetric. $F$ is a smooth map. $p \in \mathbb{R}_{+}$controls the size of the initial datum. The initial datum is oscillatory.

For the sake of simplicity, let us suppose that the nonlinearity $F$ is homogeneous of order $q\left(F(U) \sim U^{q}\right)$. Classical existence theorems for (1.3)-(1.4) give solutions defined on time intervals of the form $\left[0, t_{0} \varepsilon^{(1-q) p}\right]$. One can address the question of the behaviour of the solutions as $\varepsilon \rightarrow 0$. The case where $p=0$ is called the geometrical optics regime. In this regime, the solution $U^{\varepsilon}$ is described by

$$
U^{\varepsilon} \sim e^{i(k x-\omega t) / \varepsilon} U(t, x)+c . c .
$$

where $\omega$ and $k$ satisfy the dispersion relation

$$
\operatorname{det}\left(-\omega+\mathcal{A}(k)+\mathcal{L}_{0} / i\right)=0
$$

and $U$ satisfy a nonlinear transport equation

$$
\left(\partial_{t}+\omega^{\prime}(k) \cdot \partial_{x}\right) U=F_{1}(U)
$$

where $F_{1}$ can be expressed in terms on the Taylor expansion of $F$. For smaller solutions, the lifetime is longer and on a longer time scale, dispersive effects have to be considered: for $p=1 / q-1$, we have

$$
U^{\varepsilon} \sim \varepsilon^{1 /(p-1)}\left(e^{i(k x-\omega t) / \varepsilon} V\left(\varepsilon t, x-\omega^{\prime}(k) t\right)+\text { c.c. }\right),
$$


where $V(t, x)$ satisfies:

$$
i \partial_{\tau} V+\frac{1}{2} \omega^{\prime \prime}(k)\left(\partial_{x}, \partial_{x}\right) V=G(V)
$$

This is a classical result of Joly, Métivier and Rauch [9]. In both cases, the approximations (1.5) and (1.6) are justified by error estimates. However, in many physical cases, the nonlinearity $F_{1}$ and $G$ in the limit equations vanish because of algebraic properties of the systems. This property is called transparency by Joly, Métivier and Rauch in [10]. In the physics literature, one can however find expansions giving nonlinear models. The way these models are obtained is the following. In the case $p=0$, one seeks solutions living on $\left[0, t_{0} / \varepsilon\right]$, that is on a diffractive time scale. One then obtains Davey-Stewartson-type systems [4]. For geometrical optics time scale, one has to consider large solutions of amplitude $1 / \sqrt{\varepsilon}$ in order to get nonlinear effects [10]. For diffractive time scales with rectification, one takes $p=1 / 2[6]$. In all these cases, under the transparency hypothesis, a formal WKB expansion is possible. But in order to prove error estimates of these expansions, the transparency hypothesis is not sufficient (except for dissipative cases, see [5]). Joly, Métivier and Rauch proposed a subclass of systems for which stronger transparency hypothesis can be made, which allow to obtain satisfying error estimates. This structure is inspired from the physical Maxwell-Bloch systems. It is used in [4] and [6].

These systems have the form

$$
\left\{\begin{aligned}
L^{\varepsilon}\left(\partial_{t}, \partial_{x}\right) u & =\left(\partial_{t}+A\left(\partial_{x}\right)+\frac{1}{\varepsilon} L_{0}\right) u=f(u, m) \\
M^{\varepsilon}\left(\partial_{t}, \partial_{x}\right) m & =\left(\partial_{t}+M\left(\partial_{x}\right)+\frac{1}{\varepsilon} M_{0}\right) m=g(u, u)
\end{aligned}\right.
$$

where the operators $L^{\varepsilon}$ an $M^{\varepsilon}$ have the same structure as $\mathcal{L}^{\varepsilon}$ in (1.3). The idea is to look for solutions to (1.7) for which $m$ has a normal size (in comparison with the lifetime of the solution), that is for example $m$ of size $\varepsilon$ on $\left[0, t_{0} / \varepsilon\right]$, while $u$ is bigger (for example $u$ of size $O(1)$ on the same interval). Therefore, 
the first equation of (1.7) can be handled in a straightforward manner and the problem is to solve the second equation in (1.7).

After a rescaling, this is equivalent to considering

$$
\left\{\begin{aligned}
L^{\varepsilon}\left(\partial_{t}, \partial_{x}\right) u & =d(\varepsilon) f(u, m) \\
M^{\varepsilon}\left(\partial_{t}, \partial_{x}\right) m & =\frac{1}{c(\varepsilon)} g(u, u)
\end{aligned}\right.
$$

where $c(\varepsilon) \rightarrow 0$ as $\varepsilon \rightarrow 0$, and $u$ and $m$ have size $O(1)$.

In [10], one has $c(\varepsilon)=\varepsilon$ and $d(\varepsilon)=1$ on a time interval $\left[0, t_{0}\right]$. In [4], one has $d(\varepsilon)=\varepsilon, c(\varepsilon)=\varepsilon$ and the time is $O(1 / \varepsilon)$. In [6], one has $d(\varepsilon)=\varepsilon, c(\varepsilon)=1$ and the time is $O(1 / \varepsilon)$. These scaling are not equivalent, see remark 1.15.

Here our goal is to justify the Zakharov equations. In this view, the appropriate scaling is $d(\varepsilon)=1, c(\varepsilon)=\sqrt{\varepsilon}$ and $M^{\varepsilon}$ has the form

$$
M^{\varepsilon}\left(\partial_{t}, \partial_{x}\right)=\partial_{t}+\sqrt{\varepsilon} B\left(\partial_{x}\right)
$$

that is we take $M_{0}=0$ and we assume that the acoustic velocity is small. This is reasonable in the physical context of plasma waves. Note that thanks to the fact that the acoustic velocity is small, the transparency Assumption 1.7 needed for the proof of the error estimate is weaker than the hypothesis in $[10]$.

This scaling is actually not completely convenient in order to derive the Zakharov system and we will therefore consider the new spatial variable $X=$ $x / \sqrt{\varepsilon}$, that we still denote by $x$. The typical system that we will consider in this paper will therefore be

$$
\left\{\begin{aligned}
\left(\partial_{t}+\frac{1}{\sqrt{\varepsilon}} A\left(\partial_{x}\right)+\frac{L_{0}}{\varepsilon}\right) u & =f(u, m), \\
\left(\partial_{t}+B\left(\partial_{x}\right)\right) m & =\frac{1}{\sqrt{\varepsilon}} g(u, u) .
\end{aligned}\right.
$$


In the next section, we present examples of systems on which our results apply.

\subsection{Examples}

A multidimensional Klein-Gordon-wave system. The following system is directly obtained from the bifluid Euler-Maxwell system (see section 2.1):

$$
\left\{\begin{aligned}
\left(\partial_{t}^{2}-\frac{1}{\varepsilon} \Delta+\frac{1}{\varepsilon^{2}}\right) E & =\quad n E \\
\left(\partial_{t}^{2}-\Delta\right) n & =\Delta|E|^{2} .
\end{aligned}\right.
$$

It will be shown in section 2.1 that up to a change of unknowns this system can be considered as being of the form (1.9). Our main result (Theorem 1.9) therefore applies: Let $E^{0}, n_{0}^{0}, n_{1}^{0} \in H^{\sigma}$ with $\sigma$ large enough. Let $F$ and $N$ be the solution of the Zakharov system

$$
\left\{\begin{aligned}
&-2 i \partial_{t} F+\Delta F=\quad N F \\
& \partial_{t}^{2} N-\Delta N=\Delta|F|^{2}
\end{aligned}\right.
$$

with $F(0)=E^{0}, N(0)=n_{0}^{0}, \partial_{t} N(0)=n_{1}^{0}$. $(F, N)$ is defined on $\left[0, t_{0}\right]$, with $t_{0}>0$. There exists $E, n$ solution to (1.10) defined over $\left[0, t_{0}\right]$, with the same initial datum as $F, N$, and such that

$$
\| E-\left(F e^{-i t / \varepsilon}+\text { c.c. }\right)\|+\| n-N \|=O(\sqrt{\varepsilon})
$$

where $\|\cdot\|$ is the $L^{\infty}\left(\left[0, t_{0}\right] \times \mathbb{R}^{3}\right)$ norm.

A quasilinear, one-dimensional Klein-Gordon-wave system. Consider the system

$$
\left\{\begin{aligned}
\partial_{t}\left(\begin{array}{c}
u \\
v
\end{array}\right)+\frac{1}{\sqrt{\varepsilon}} \partial_{x}\left(\begin{array}{c}
v \\
u
\end{array}\right)+\frac{1}{\varepsilon}\left(\begin{array}{c}
-v \\
u
\end{array}\right) & =n\left(\begin{array}{c}
v \\
u
\end{array}\right), \\
\left(\partial_{t}^{2}-\partial_{x}^{2}\right) n & =\partial_{x}^{2}\left(u^{2}+v^{2}\right) .
\end{aligned}\right.
$$


A local, nonlinear change of variables (section 2.2) transforms (1.12) into a system of the form (1.7). This is a change of variables that transforms the quasilinear term in (1.12) into a semilinear term. For this system, the result is similar to the result for the multidimensional Klein-Gordon system (1.10).

A quasilinear, two-dimensional Klein-Gordon-wave system. The two-dimensional version of (1.12) is

$$
\left\{\begin{aligned}
\partial_{t}\left(\begin{array}{l}
u \\
v
\end{array}\right)+\frac{1}{\sqrt{\varepsilon}} \partial_{x}\left(\begin{array}{c}
-u \\
v
\end{array}\right)+\frac{1}{\sqrt{\varepsilon}} \partial_{y}\left(\begin{array}{l}
v \\
u
\end{array}\right)+\frac{1}{\varepsilon}\left(\begin{array}{c}
-v \\
u
\end{array}\right) & =n\left(\begin{array}{l}
v \\
u
\end{array}\right), \\
\left(\partial_{t}^{2}-\Delta_{x, y}\right) n & =\Delta_{x, y}\left(u^{2}+v^{2}\right) .
\end{aligned}\right.
$$

This system cannot be transformed into a system of the form (1.9). We show in section 2.4.1 how the quasilinear coupling term has to be modified to allow such a tranformation.

\subsection{Assumptions and results}

Consider the symmetric hyperbolic operators

$$
\begin{aligned}
L^{\varepsilon}\left(\partial_{t}, \partial_{x}\right) & :=\partial_{t}+\frac{1}{c(\varepsilon)} A\left(\partial_{x}\right)+L_{0} / \varepsilon=\partial_{t}+\frac{1}{c(\varepsilon)} \sum_{j=1}^{d} A_{j} \partial_{j}+L_{0} / \varepsilon, \\
M\left(\partial_{t}, \partial_{x}\right) & :=\partial_{t}+B\left(\partial_{x}\right)=\partial_{t}+\sum_{j=1}^{d} B_{j} \partial_{j} .
\end{aligned}
$$

The $A_{j}$ 's are hermitian $N \times N$ matrices; the $B_{j}$ are hermitian $N_{1} \times N_{1}$ matrices. $L_{0}$ is a skewhermitian $N \times N$ matrix. Let $f$ be a bilinear map $\mathbb{C}^{N} \times \mathbb{C}^{N_{1}} \rightarrow \mathbb{C}^{N}$, 
and let $g$ be a bilinear map: $\mathbb{C}^{N} \times \mathbb{C}^{N} \rightarrow \mathbb{C}^{N_{1}}$. Let $U=(u, m) \in \mathbb{C}^{N \times N_{1}}$ and

$$
\begin{aligned}
\mathcal{L}^{\varepsilon}(\partial) & :=\left(\begin{array}{cc}
L^{\varepsilon}(\partial) & 0 \\
0 & M^{\varepsilon}(\partial)
\end{array}\right) \\
\mathcal{C}(U, U) & :=\left(\begin{array}{c}
f(u, m) \\
0
\end{array}\right), \quad \mathcal{B}(U, U):=\left(\begin{array}{c}
0 \\
g(u, u)
\end{array}\right) .
\end{aligned}
$$

The system we consider is

$$
\mathcal{L}^{\varepsilon}\left(\partial_{t, x}\right) U^{\varepsilon}=\frac{1}{c(\varepsilon)} \mathcal{B}\left(U^{\varepsilon}, U^{\varepsilon}\right)+\mathcal{C}\left(U^{\varepsilon}, U^{\varepsilon}\right)
$$

With the above notations, it has the form (1.8) with $d(\varepsilon)=1$.

We are interested in the regime $c(\varepsilon)=\sqrt{\varepsilon}$. For $c(\varepsilon)=\sqrt{\varepsilon}$, classical results [18] provide the existence of a unique regular solution to the Cauchy problem for (1.14) with an initial datum of size $O(1)$ (with respect to $\varepsilon$ ) in $H^{\sigma}\left(\mathbb{R}^{d}\right)$, for $\sigma>N / 2$. The existence time is a priori only $O(\sqrt{\varepsilon})$.

Definition 1.1 (approximate solutions) Given an initial datum $U^{0}(x) \in$ $H^{\infty}\left(\mathbb{R}_{x}^{d}\right)$, we say that $U_{a}^{\varepsilon}$ defined over $\left[0, t^{*}\right.$ [ is an approximate solution at order $k$ to (1.14) with initial value $U^{0}$ when

$$
\left\{\begin{array}{c}
\mathcal{L}^{\varepsilon}\left(\partial_{t, x}\right) U_{a}^{\varepsilon}-\frac{1}{\sqrt{\varepsilon}} \mathcal{B}\left(U_{a}^{\varepsilon}, U_{a}^{\varepsilon}\right)-\mathcal{C}\left(U_{a}^{\varepsilon}, U_{a}^{\varepsilon}\right)=\varepsilon^{k} R^{\varepsilon} \\
U_{a}^{\varepsilon}(0)=U^{0}
\end{array}\right.
$$

where $R^{\varepsilon} \rightarrow 0$ as $\varepsilon \rightarrow 0$ in $L^{\infty}\left(\left[0, t_{0}\right] \times \mathbb{R}^{d}\right)$, for all $0 \leq t_{0}<t^{*}$.

Note that we will actually use a slightly stronger notion of approximate solutions, via the representation by profiles.

Definition 1.2 (stability) An approximate solution $U_{a}^{\varepsilon}$ at order $k$ with initial value $U^{0}$ is said to be stable over some time interval $\left[0, t^{* *}\right.$ [ independent of 
$\varepsilon$, when for all family of maps $H^{\varepsilon}(x) \in H^{\infty}\left(\mathbb{R}_{x}^{d}\right)$, such that $H^{\varepsilon} \rightarrow 0$ as $\varepsilon \rightarrow 0$ in $H^{\infty}\left(\mathbb{R}_{x}^{d}\right)$, the solution $U^{\varepsilon}$ to the Cauchy problem for (1.14) with the initial datum $U^{\varepsilon}(0)=U^{0}+\varepsilon^{k} H^{\varepsilon}$ satisfies

$$
\left\|U^{\varepsilon}-U_{a}^{\varepsilon}\right\|_{L^{\infty}\left(\left[0, t_{0}\right] \times \mathbb{R}^{d}\right)}=o(1)
$$

as $\varepsilon \rightarrow 0$, for all $0 \leq t_{0}<t^{* *}$.

Remark 1.3 - Note that this notion of stability is weaker than the one used in [2].

- The error estimate in (1.15) can be made precise, depending on the precision of the approximate solution and the initial data (see further, after the statement of Theorem 1.9).

For $\beta=(\omega, k) \in \mathbb{R} \times \mathbb{R}^{d}$, introduce the notations:

$$
L^{\varepsilon}\left(\beta \partial_{\theta}\right):=-\omega \partial_{\theta}+\frac{1}{\sqrt{\varepsilon}} A\left(k \partial_{\theta}\right)+\frac{1}{i} L_{0}, \quad M\left(\beta \partial_{\theta}\right):=-\omega \partial_{\theta}+M\left(k \partial_{\theta}\right) .
$$

The characteristic varieties of $L^{\varepsilon}$ and $M^{\varepsilon}$ are defined as

$$
\begin{aligned}
& \text { Char } L^{\varepsilon}:=\left\{\beta \in \mathbb{R} \times \mathbb{R}^{d}, \operatorname{det} L^{\varepsilon}(\beta)=0\right\} \\
& \text { Char } M:=\left\{\beta \in \mathbb{R} \times \mathbb{R}^{d}, \operatorname{det} M(\beta)=0\right\}
\end{aligned}
$$

For a characteristic phase $\beta=(\omega, k) \in$ Char $L^{\varepsilon}$, one denotes by $P(\beta)$ the orthogonal projector onto the Kernel of $-\omega+A(k) / \sqrt{\varepsilon}+L_{0} / i$, and by $\mathcal{R}$ the set of all integers $p \in \mathbb{Z}$, such that $p \beta$ is characteristic $\left(p \beta \in\right.$ Char $\left.L^{\varepsilon}\right)$. $P$ and $\mathcal{R}$ actually depends on $\varepsilon$, as the matrix $L^{\varepsilon}(\beta)$ depends on $\varepsilon$ (see (1.16)). For fixed $\varepsilon$ and for dispersive equations $\left(L_{0} \neq 0\right)$, it is reasonable to assume that $\mathcal{R}$ is a finite set (this is the "strong finiteness assumption" of [9]), and we will do so. 
Note that for $\beta=(\omega, 0)$, the matrix $L^{\varepsilon}(p \beta)$ and the associated projector $P(p \beta)$ and characteristic harmonic set $\mathcal{R}$ actually do not depend on $\varepsilon$. Such a characteristic phase is considered in section 3 and in Theorem 1.9 (but in Theorem 1.8, we can allow more general phases $(\omega, k)$ - see below).

For a characteristic phase $\beta^{\prime}=\left(\omega^{\prime}, k^{\prime}\right) \in$ Char $M$, one denotes by $Q\left(\beta^{\prime}\right)$ the orthogonal projector onto the Kernel of $-\omega+B\left(k^{\prime}\right)$.

We need some kind of regularity for the phases $p \beta \in$ Char $L^{\varepsilon}, p \in \mathcal{R}$, but we want to allow these points to be critical (in the sense of definition 1.4 of [26]; this means that we want to allow several branches of the variety to intersect at $p \beta)$ ) as this is the case for the Euler-Maxwell equations (see the description of the characteristic variety of the Euler-Maxwell equations in [27]). It is convenient to assume that Char $L^{\varepsilon}$ satisfies an axisymmetry property. This means that we assume in the following that the eigenvalues of $A(k) \sqrt{\varepsilon}+L_{0} / i$ depend only on $k$ only through $|k|$. With this assumption, Butler's theorem $[16,26]$ implies that Char $L^{\varepsilon}$ is parameterized by smooth maps. This assumption is satisfied by the physical examples we give in this paper and by the Euler-Maxwell equations.

We will need some regularity for the eigenprojectors of the matrices $B(k)$ as well. Again, it suffices to assume that the eigenvalues of $B(k)$ depend only on $|k|$, an assumption satisfied by our model systems.

In section 3, we fix a characteristic phase $\beta=(\omega, 0) \in$ Char $L^{\varepsilon}$ and we construct approximate WKB solutions in the variables $t, x, \theta=\omega t / \varepsilon$. We call "profiles" maps depending on the variables $t, x, \theta$ which are periodic in $\theta$. We make in section 3 the following "weak transparency" assumptions: 
Assumption 1.4 For all $p \in \mathcal{R}-\{0\}$, for all $u, u^{\prime} \in \mathbb{C}^{N}$, one has

$$
g\left(P(p \beta) u, P(-p \beta) u^{\prime}\right)=0 .
$$

Assumption 1.5 For all $p \in \mathcal{R}-\{0\}, p \beta$ is a local extremum of every branch of Char $L^{\varepsilon}$ that passes at $p \beta$.

Assumption 1.4 is a necessary condition for the construction of WKB solutions of high-amplitude (this is obvious from the WKB modulation equations, specifically (3.44)). Assumption 1.5 will ensure that the equations for the profiles of the WKB expansions are well-posed (see remark 3.1 about the well-posedness of the limit system). Assumption 1.5 precisely means that, for a given $p \in \mathcal{R}$, if $k^{\prime} \mapsto \omega_{1}\left(k^{\prime}\right), \ldots, k^{\prime} \mapsto \omega_{s}\left(k^{\prime}\right)$ are the eigenvalues of $\frac{1}{\sqrt{\varepsilon}} A\left(k^{\prime}\right)+L_{0} / i$ that satisfy $\omega_{j}(k)=p \omega$, then $\omega_{j}^{\prime}(k)=0$ for all $k$ (where ' denotes differentiation with respect to $k \in \mathbb{R}^{d}$ - the eigenvalues are analytic thanks to the axisymmetry assumption).

Proposition 1.6 Under Assumptions 1.4 and 1.5, for any initial datum $U^{0}(x) \in$ $H^{\infty}\left(\mathbb{R}_{x}^{d}\right)$, there exists $t^{*}>0$ and an approximate solution $U_{\text {app }}^{\varepsilon}$ at order 1 defined over $\left[0, t^{*}\left[. U_{a p p}^{\varepsilon}\right.\right.$ is represented by a profile $\mathbf{U}_{a p p}^{\varepsilon}$ and has the form of a WKB expansion

$$
\begin{aligned}
U_{a p p}^{\varepsilon}(t, x) & =\left[\mathbf{U}_{a p p}^{\varepsilon}(t, x, \theta)\right]_{\theta=\omega t / \varepsilon} \\
& =\left[\left(\mathbf{U}_{0}+\sqrt{\varepsilon} \mathbf{U}_{1}+\varepsilon \mathbf{U}_{2}\right)(t, x, \theta)\right]_{\theta=\omega t / \varepsilon} .
\end{aligned}
$$

$\mathbf{U}_{0}=(u, m)$ is solution of the vector Zakharov-type system (3.46)-(3.47).

The question of the global existence for the Zakharov equations in several space dimensions is still open, so we only have $t^{*}<\infty$ a priori. 
In section 4 , we fix a characteristic phase $\beta=(\omega, k) \in$ Char $L^{\varepsilon}$, where $k$ is possibly nonzero, and we prove an energy estimate for the linearized equations in the variable $t, x, \theta=(k \cdot x-\omega t) / \varepsilon$ under the following "approximate linear transparency" assumption (where the projectors $P$ and $Q$ were introduced shortly after Remark 1.3):

Assumption 1.7 There exists $\varepsilon_{0}>0$ and $C>0$ such that for $\left.\left.\varepsilon \in\right] 0, \varepsilon_{0}\right]$, for all $\xi=(\tau, \eta) \in$ Char $L^{\varepsilon}$, for all $\xi^{\prime}=\left(\tau^{\prime}, \eta+p k\right) \in$ Char $M^{\varepsilon}$, for all $p \in \mathcal{R}$, if

$$
\left|\tau^{\prime}-(\tau+p \omega)\right| \leq \sqrt{\varepsilon}
$$

then for all $u, u^{\prime} \in \mathbb{C}^{N}$,

$$
\min \left(1, \frac{\varepsilon}{\left|\tau^{\prime}-(\tau+p \omega)\right|}\right)\left|Q\left(\xi^{\prime}\right) g\left(P(p \beta) u, P(\xi) u^{\prime}\right)\right| \leq C \sqrt{\varepsilon}|u|\left|u^{\prime}\right| .
$$

We check that Assumption 1.7 is satisfied by our model systems in section 2.4.1. This assumption is weaker than the corresponding assumption of Joly, Métivier and Rauch [10], which is

$$
\left|Q\left(\xi^{\prime}\right) g\left(P(p \beta) u, P(\xi) u^{\prime}\right)\right| \leq C|u|\left|u^{\prime}\right|\left|\tau^{\prime}-(\tau+p \omega)\right| .
$$

For example, when $\left|\tau^{\prime}-(\tau+p \omega)\right|=O(\sqrt{\varepsilon})$, we allow the interaction coefficient

$$
\left|Q\left(\xi^{\prime}\right) g\left(P(p \beta) u, P(\xi) u^{\prime}\right)\right|
$$

to be of size $O(1)$ whereas (1.19) implies that it is of size $O(\sqrt{\varepsilon})$. Note, however, that the regime we consider is less singular than the regime under consideration in [10] - see remark 1.13.

Assumption 1.7 is the key of the stability proof of section 4.1, as it provides a control of the resonances (constructive interactions of waves). The interaction is through $g$, the semilinear source term. The (generalized) resonance 
condition is $\Phi=\left|\tau^{\prime}-(\tau+p \omega)\right| \leq \sqrt{\varepsilon}$. We assume that the corresponding interaction coefficient $C=\left|Q\left(\xi^{\prime}\right) g\left(P(p \beta) u, P(\xi) u^{\prime}\right)\right|$ is small (compare with Assumption 2.5 of [10] where the authors assume that the interaction coefficients vanish - transparency - at the resonances). After changes of variables, it appears in the course of the stability proof (section 4.1) that our goal is to find uniform bounds (with respect to $\varepsilon$ ) for singular integrals of the type

$$
\frac{1}{\sqrt{\varepsilon}} \int_{0}^{t} \exp \left(\frac{i t \Phi}{\varepsilon}\right) C d t^{\prime}
$$

This amounts, by non-stationnary phase arguments, to find a uniform bound for the ratio

$$
\left|\frac{C / \sqrt{\varepsilon}}{\Phi / \varepsilon}\right|
$$

Assumption 1.7 precisely provides such a bound. The existence of such a bound can be seen as a transparency property (in the sense of Joly, Métivier and Rauch [10]) of our Klein-Gorond-waves systems.

We will make use of this assumption in the following context. Let $t^{*}>0$ and let $\mathbf{U}_{a}^{\varepsilon}=\left(\mathbf{u}_{a}^{\varepsilon}, \mathbf{m}_{a}^{\varepsilon}\right)$ be a family of profiles in the variables $t, x, \theta$, defined over $\left[0, t^{*}\left[\right.\right.$, uniformly bounded in $W^{1, \infty}\left(\left[0, t_{0}\right], H^{\sigma}\left(\mathbb{R}_{x}^{d} \times \mathbb{T}\right)\right)$, for all $\sigma \in \mathbb{R}$ and all $0 \leq t_{0}<t^{*}$. There exists $C$ independent of $\varepsilon$ such that $\left\|\mathbf{U}_{a}^{\varepsilon}, \partial_{t} \mathbf{U}_{a}^{\varepsilon}\right\|_{L^{\infty}\left(\left[0, t_{0}\right], H^{\sigma}\right)} \leq$ $C\left(\sigma, t_{0}\right)$. We also suppose that for all $p$,

$$
(1-P(p \beta)) u_{a, p}^{\varepsilon}=\sqrt{\varepsilon} \tilde{u}_{a, p}^{\varepsilon},
$$

where $\tilde{\mathbf{u}}_{a}^{\varepsilon} \in W^{1, \infty}\left(\left[0, t_{0}\right], H^{\sigma}\left(\mathbb{R}_{x}^{d} \times \mathbb{T}\right)\right.$, for all $\sigma \in \mathbb{R}$ and all $0 \leq t_{0}<t^{*}$ (where $u_{p}$ is the $p$ th Fourier coefficient of the profile $\mathbf{u}$ ). Note that (1.21) is satisfied by the approximate solution constructed in section 3, see in particular equation (3.43). In the profile variables $t, x, \theta$, the leading term of the operator linearized around $U_{a}^{\varepsilon}$ is

$$
\mathcal{L}_{1}^{\varepsilon}\left(\mathbf{U}_{a}^{\varepsilon}, \partial_{t}, \partial_{x}, \frac{\beta}{\varepsilon} \partial_{\theta}\right):=\left(\begin{array}{cc}
L^{\varepsilon}\left(\partial_{t}, \partial_{x}\right)+\frac{1}{\varepsilon} L^{\varepsilon}\left(\beta \partial_{\theta}\right) & 0 \\
-\frac{1}{\sqrt{\varepsilon}} g\left(\mathbf{u}_{a}^{\varepsilon}\right) & M\left(\partial_{t}, \partial_{x}\right)+\frac{1}{\varepsilon} M\left(\beta \partial_{\theta}\right)
\end{array}\right)
$$


where $g\left(u_{a}\right) u:=g\left(u_{a}, u\right)+g\left(u, u_{a}\right)$, and where the notations $L^{\varepsilon}(\beta)$ and $M(\beta)$ were introduced in (1.16). With these notations, the following theorem holds:

Theorem 1.8 Consider a profile $\mathbf{U}_{a}^{\varepsilon}$ defined over $\left[0, t^{*}[\right.$, uniformly bounded in $W^{1, \infty}\left(\left[0, t_{0}\right], H^{\sigma}\left(\mathbb{R}_{x}^{d} \times \mathbb{T}\right)\right)$, for all $\sigma \in \mathbb{R}$ and all $0 \leq t_{0}<t^{*}$, and satisfying condition (1.21). Under Assumption 1.7, where $\beta=(\omega, k)$, for sufficiently large $\sigma$, for all $0 \leq t_{0}<t^{*}$, there exists $C\left(\sigma, t_{0}\right)>0$ such that for all profile $\mathbf{U} \in C^{0}\left(\left[0, t_{0}\right], H^{\sigma+1}\left(\mathbb{R}_{x}^{d} \times \mathbb{T}\right)\right) \cap C^{1}\left(\left[0, t_{0}\right], H^{\sigma}\left(\mathbb{R}_{x}^{d} \times \mathbb{T}\right)\right)$, for all $0 \leq t \leq t_{0}$, the energy estimate holds

$$
\|\mathbf{U}(t)\|_{H^{\sigma}\left(\mathbb{R}_{x}^{d} \times \mathbb{T}\right)} \leq\|U(0)\|_{H^{\sigma}\left(\mathbb{R}^{d}\right)}+C \int_{0}^{t}\left\|\mathcal{L}_{1}^{\varepsilon}\left(\mathbf{U}_{a}^{\varepsilon}, \partial_{t}, \partial_{x}, \frac{\beta}{\varepsilon} \partial_{\theta}\right) \mathbf{U}\left(t^{\prime}\right)\right\|_{H^{\sigma}\left(\mathbb{R}_{x}^{d} \times \mathbb{T}\right)} d t^{\prime} .
$$

We will make use of this theorem with $\mathbf{U}_{a}^{\varepsilon}=\mathbf{U}_{a p p}^{\varepsilon}$, the profile representing the approximate solution constructed in section 3. With Proposition 1.14, Theorem 1.8 implies our main result:

Theorem 1.9 Under Assumptions 1.4, 1.5, 1.7, where $\beta=(\omega, 0)$, the approximate solution $U_{a p p}^{\varepsilon}$ is stable over its interval of existence $\left[0, t^{*}[\right.$.

In this theorem, we have to restrict to a characteristic phase $\beta=(\omega, 0) \in$ Char $L^{\varepsilon}$, that is with $k=0$, in order to ensure well-posedness of the limit system (3.46)-(3.47) (see remark 3.1).

In (1.15), the error estimate depends on the order of the approximate solution and on the size of the perturbation of the initial data (see the proof of Proposition 1.14). In particular, for approximate solutions at order 1 with the same initial datum as the exact solution, the error estimate is $O(\sqrt{\varepsilon})$. 
Remark 1.10 The extension of Theorem 1.9 to the full, quasilinear EulerMmaxwell equations is a very interesting direction for future work. The Fourier analysis of the proof of Theorem 1.9 cannot be directly applied to the EulerMaxwell equations. Indeed, for quasilinear systems, the perturbation equation has varying coefficients. However, one can expect the Klein-Gordon-waves system to be a good approximation of Euler-Maxwell for low frequencies, and Assumption 1.7 to hold for the Euler-Maxwell system for low frequencies. For high-frequencies, the convection terms in the Euler equations, that were essentially neglected in the derivation of the Klein-Gordon-waves systems (see section 2.1), are not negligible, and may create additional resonances.

In section 2.3, we consider quasilinear systems of the form:

$$
\left\{\begin{aligned}
L^{\varepsilon}\left(\partial_{t}, \partial_{x}\right) u & =f(u, m) \\
M\left(\partial_{t}, \partial_{x}\right) m & =\partial_{x} g(u, u)
\end{aligned}\right.
$$

where $L^{\varepsilon}$ and $M^{\varepsilon}$ have the same form as above. We state and discuss a "strong transparency assumption at infinity":

Assumption 1.11 There exists $C>0$ such that for all $u, u^{\prime} \in \mathbb{C}^{n}$, for all $k, l, l^{\prime}$ and for all $\eta, \eta^{\prime} \in \mathbb{R}^{d}$, with $\eta+\eta^{\prime} \neq 0$,

$$
\left|Q_{l}\left(\eta+\eta^{\prime}\right) g\left(P_{k}(\eta) u, P_{k^{\prime}}\left(\eta^{\prime}\right) u^{\prime}\right)\right| \leq C\left|c(\varepsilon) \mu_{l}\left(\eta+\eta^{\prime}\right)-\lambda_{k}(\eta)-\lambda_{k^{\prime}}\left(\eta^{\prime}\right)\right||u|\left|u^{\prime}\right|
$$

where $P_{k}, \lambda_{k}$ and $Q_{l}, \mu_{l}$ are elements of the spectral decomposition of $A$ and $B$ :

$$
A(\eta)=\sum_{k} \lambda_{k}(\eta) Q_{k}(\eta), \quad B(\eta)=\sum_{l} \mu_{l}(\eta) Q_{l}(\eta) .
$$

We prove the following proposition:

Proposition 1.12 Under assumption 1.11, there exists bounded families of bilinear maps $\left.\left.S^{\varepsilon}, h^{\varepsilon}: H^{\sigma} \times H^{\sigma} \rightarrow H^{\sigma}, \varepsilon \in\right] 0,1\right]$, such that the change of 
variables

$$
\left(\begin{array}{c}
u \\
m
\end{array}\right)=\left(\begin{array}{c}
\tilde{u} \\
\tilde{m}+S^{\varepsilon}(\tilde{u}, \tilde{u})
\end{array}\right),
$$

turns (1.23) into

$$
\left\{\begin{aligned}
L^{\varepsilon}(\partial) \tilde{u} & =f\left(\tilde{u}, \tilde{m}+S^{\varepsilon}(\tilde{u}, \tilde{u})\right), \\
M^{\varepsilon}(\partial) \tilde{m} & =\frac{c(\varepsilon)}{\varepsilon} h^{\varepsilon}(\tilde{u}, \tilde{u})+\tilde{f}^{\varepsilon}(\tilde{u}, \tilde{m}),
\end{aligned}\right.
$$

where $\left\{\tilde{f}^{\varepsilon}\right\}_{\varepsilon \in] 0,1]}$ is a bounded family of polynomial maps.

It is interesting to compare the transparency assumptions 1.4, 1.5, 1.7, 1.11 with assumptions $2.1,2.2,2.5$ and 2.10 of [10]:

- Assumptions 1.4 is the same as Assmption 2.1 in [10]. Both are necessary conditions for the existence of high-amplitude WKB solutions.

- Assumption 1.5 is comparable to Assumption 2.2 in [10] as it ensures that the profile equations are well-posed.

- Assumption 1.7 is weaker than Assumption 2.5 of [10]. We do not suppose that the interaction coefficients vanish at the resonances. We only suppose that we have a certain control of the ratio (1.20).

- Assumption 1.11 is comparable to Assumption 2.10 of [10] as it implies a conjugation result. Here we conjugate quasilinear and semilinear systems. Joly, Métivier and Rauch used their Assumption 2.10 to conjugate their Maxwell-Bloch systems to systems for which weakly nonlinear results apply. They proved that Assumption 2.10 is stronger than Assumption 2.5. As Assumption 2.5 is actually not satisfied by our model systems, we cannot expect such an implication. 
Remark 1.13 Note that the nonlinear regime in consideration in this paper is a regime of strong oscillations (oscillations fortes in the sense of Cheverry, Guès and Métivier [2]). That is, we look for profiles of size $O(1)$, that oscillate with characteristic frequencies of size $O(1 / \varepsilon)$, that are defined over a time interval $O(1)$, and that solve systems with a bilinear singular term with a prefactor $O(1 / \sqrt{\varepsilon})$. The regime that Joly, Métivier and Rauch considered in [10] was more singular (same context, but singular prefactor $O(1 / \varepsilon)$ ). This explains why we can prove stability in a less transparent context than in [10].

\subsection{Linear and nonlinear stability}

We say that the linearized equations are stable over $\left[0, t^{* *}[\right.$, when an estimate like $(1.22)$ is available over $\left[0, t^{* *}\right.$. The following proposition asserts that when the linearized equations are stable, the approximate solution is stable in the sense of definition 1.2. In other words, linear stability implies nonlinear stability in our setting.

Let $\mathbf{U}_{a}^{\varepsilon}$ be a family of profiles with a time existence $t^{*}>0$. Suppose that $\mathbf{U}_{a}^{\varepsilon}$ is an approximate solution to (1.14) in the profile variables: there exists $\mathbf{R}^{\varepsilon} \in L^{\infty}\left(\left[0, t_{0}\right], H^{\sigma}\left(\mathbb{R}^{d} \times \mathbb{T}\right)\right)$, with $\mathbf{R}^{\varepsilon} \rightarrow 0$ as $\varepsilon \rightarrow 0$, for all $0 \leq t_{0}<t^{*}$, such that

$$
\mathcal{L}^{\varepsilon}\left(\partial_{t}, \partial_{x}, \frac{\beta}{\varepsilon} \partial_{\theta}\right) \mathbf{U}_{a}^{\varepsilon}-\frac{1}{\sqrt{\varepsilon}} \mathcal{B}\left(\mathbf{U}_{a}^{\varepsilon}, \mathbf{U}_{a}^{\varepsilon}\right)-\mathcal{C}\left(\mathbf{U}_{a}^{\varepsilon}, \mathbf{U}_{a}^{\varepsilon}\right)=\varepsilon^{k} \mathbf{R}^{\varepsilon}
$$

To $\mathbf{U}_{a}^{\varepsilon}$ corresponds a family of maps $U_{a}^{\varepsilon}$ via (1.18).

Proposition 1.14 With the above notations, if $\sigma>d / 2$, then $U_{a}^{\varepsilon}$ is an approximate solution at order $k$ to (1.14) in the sense of definition 1.1. Suppose that the linearized equations are stable over $\left[0, t^{*}\left[\right.\right.$. Then if $k>1 / 2$, then $U_{a}^{\varepsilon}$ is stable over $\left[0, t^{*}\left[\right.\right.$ and if $k=1 / 2, U_{a}^{\varepsilon}$ is stable over $\left[0, t^{* *}\left[\right.\right.$, with $t^{* *} \leq t^{*}$. 
Proof: The condition $\sigma>d / 2$ ensures that the $H^{\sigma}$ bounds for the profiles give $L^{\infty}$ bounds for maps in the physical variables $t, x$. Thus $U_{a}^{\varepsilon}$ is an approximate solution. The proof of its nonlinear stability relies classically on a Taylor expansion and Gronwall's lemma. We work in the profile variables $t, x, \theta$. Classical results [18] provide a local existence and uniqueness result for the Cauchy problem for (1.14) in the variables $t, x, \theta$ and with the initial value $U^{0}+\varepsilon^{k} H^{\varepsilon}$. The time existence given by [18] is only $O(\sqrt{\varepsilon})$. We look for the exact solution $V^{\varepsilon}$ in the form of a profile which is a perturbation of $\mathbf{U}_{a}^{\varepsilon}$ :

$$
\mathbf{V}^{\varepsilon}=\mathbf{U}_{a}^{\varepsilon}+\varepsilon^{k} \mathbf{U}^{\varepsilon}
$$

We have

$$
\begin{aligned}
\varepsilon^{-k}\left(\mathcal{L}^{\varepsilon}\left(\partial_{t}, \partial_{x}, \varepsilon^{-1} \beta \partial_{\theta}\right) \mathbf{U}_{a}^{\varepsilon}\right. & \left.-\varepsilon^{-1 / 2} \mathcal{B}\left(\mathbf{U}_{a}^{\varepsilon}, \mathbf{U}_{a}^{\varepsilon}\right)\right)+\mathcal{L}_{1}^{\varepsilon}\left(\mathbf{U}_{a}^{\varepsilon}, \partial_{t}, \partial_{x}, \varepsilon^{-1} \beta \partial_{\theta}\right) \mathbf{U}^{\varepsilon} \\
& =\varepsilon^{k-1 / 2} \mathcal{B}\left(\mathbf{U}^{\varepsilon}, \mathbf{U}^{\varepsilon}\right)+\varepsilon^{-k}\left(\mathcal{C}\left(\mathbf{U}_{a}^{\varepsilon}+\varepsilon^{k} \mathbf{U}^{\varepsilon}\right)-\mathcal{C}\left(\mathbf{U}_{a}^{\varepsilon}\right)\right),
\end{aligned}
$$

that is

$\mathcal{L}_{1}^{\varepsilon}\left(\mathbf{U}_{a}^{\varepsilon}, \partial_{t}, \partial_{x}, \varepsilon^{-1} \beta \partial_{\theta}\right) \mathbf{U}^{\varepsilon}=-\mathbf{R}^{\varepsilon}+\varepsilon^{k-1 / 2} \mathcal{B}\left(\mathbf{U}^{\varepsilon}, \mathbf{U}^{\varepsilon}\right)+\varepsilon^{-k}\left(\mathcal{C}\left(\mathbf{U}_{a}^{\varepsilon}+\varepsilon^{k} \mathbf{U}\right)-\mathcal{C}\left(\mathbf{U}_{a}^{\varepsilon}\right)\right)$

Then (1.22) implies

$$
\begin{aligned}
\left\|\mathbf{U}^{\varepsilon}(t)\right\|_{H^{\sigma}} & \leq \varepsilon^{-k}\left(\left\|V^{\varepsilon}(0)-U_{a}^{\varepsilon}(0)\right\|_{H^{\sigma}}+C t_{0}\left\|\mathbf{R}^{\varepsilon}\right\|_{L^{\infty}\left(\left[0, t_{0}\right], H^{\sigma}\right)}\right) \\
& +C \int_{0}^{t}\left(\varepsilon^{k-1 / 2}\left\|\mathbf{U}^{\varepsilon}\left(t^{\prime}\right)\right\|_{H^{\sigma}}^{2}+\left\|\mathbf{U}_{a}^{\varepsilon}\left(t^{\prime}\right)\right\|_{H^{\sigma}}\left\|\mathbf{U}^{\varepsilon}\left(t^{\prime}\right)\right\|_{H^{\sigma}}\right) d t^{\prime} .
\end{aligned}
$$

If $k>1 / 2$, then Gronwall's lemma implies the convergence of $\mathbf{U}^{\varepsilon}$ in $H^{\sigma}\left(\mathbb{R}^{d} \times\right.$ $\mathbb{T}))$ uniformly in $t$ over any compact interval $\left[0, t_{0}\right], t_{0}<t^{*}$. If $k=1 / 2$, then Picard iterates for (1.26) provide a time existence $t^{* *}$ for $\mathbf{U}^{\varepsilon}$ which is a priori smaller than $t^{*}$, and the convergence holds over all compact interval $\left[0, t_{0}\right]$, with $t_{0}<t^{* *}$ only. Finally, the injection $H^{\sigma} \hookrightarrow L^{\infty}$ gives the asymptotic estimate of definition 1.2. 
Remark 1.15 a) High-amplitude solutions for semilinear systems are studied in [10]. Approximate solutions at all order are constructed, and an energy estimate for the linearized equations yields an error estimate valid over the time interval of the approximate solutions as in the first case of the above proposition (theorem 8.2).

b) Such a proposition does not hold for diffractive geometric optics for highamplitude solutions as considered in [6] and [4]. In these paper, an estimate of the form (1.22) is not enough to prove the error estimate. This is due to the singularities of the profile equations (due to the "long waves" asymptotics) that prevents the authors from constructing more than one corrector. As a consequence, one has to take $k=0$ in the above proof, which yields error estimates over geometric optics time intervals of size $O(1)$ only. This is why strong transparency assumptions are made in [6] and [4]. These assumptions are much stronger than assumptions providing linear stability estimates of the form (1.22) as Assumption 1.7 in this paper and Assumption 2.5 in [10].

This paper is organized as follows: in section 2.1, we derive the system (1.10) from the Euler-Maxwell system and we show how (1.10) enters the class (1.9). We give a polynomial change of variable that transforms the quasilinear model system (1.12) into a semilinear system of the form (1.7). In section 2.3 we introduce a procedure of normal forms to conjugate quasilinear systems of the form (1.23) and semilinear systems of the form (1.7). We test our transparency assumptions 1.4, 1.5, 1.7 on the systems (1.10) and (1.12) in section 2.4.1. We test the normal form procedure on the systems (1.12) and (1.13) in section 2.4.2. We perform the WKB expansion in section 3 and finally in section 4 we prove our main result, that is the stability of the WKB expansion (Theorem 1.9). 


\section{The physical models}

\subsection{Derivation of a multidimensional Klein-Gordon-wave system}

The Euler-Maxwell system describes the evolution of the electromagnetic field in a plasma. We consider a plasma as an ideal, nonhomogeneous, two-species fluid composed of electrons and ions of charge respectively $-e$ and $e$. The laser is described by the Maxwell equations; the plasma is described by the Euler equations of conservation of momentum and mass [7, 25]:

$$
\begin{aligned}
\partial_{t} B+c \nabla \times E & =0 \\
\partial_{t} E-c \nabla \times B & =4 \pi e\left(\left(n_{0}+n_{e}\right) v_{e}-\left(n_{0}+n_{i}\right) v_{i}\right), \\
\left(n_{0}+n_{e}\right)\left(\partial_{t} v_{e}+v_{e} \cdot \nabla v_{e}\right) & =-\frac{\gamma_{e} T_{e}}{m_{e}} \nabla n_{e}-\frac{e\left(n_{0}+n_{e}\right)}{m_{e}}\left(E+\frac{1}{c} v_{e} \times B\right), \\
\left(n_{0}+n_{i}\right)\left(\partial_{t} v_{i}+v_{i} \cdot \nabla v_{i}\right) & =-\frac{\gamma_{i} T_{i}}{m_{i}} \nabla n_{i}+\frac{e\left(n_{0}+n_{i}\right)}{m_{i}}\left(E+\frac{1}{c} v_{i} \times B\right), \\
\partial_{t} n_{e}+\nabla \cdot\left(\left(n_{0}+n_{e}\right) v_{e}\right) & =0 \\
\partial_{t} n_{i}+\nabla \cdot\left(\left(n_{0}+n_{i}\right) v_{i}\right) & =0 .
\end{aligned}
$$

The parameters are $m_{e}, m_{i}$ the masses of both species, $\gamma_{e}$ and $\gamma_{i}$ the specific heat ratios of both species, $T_{i}$ and $T_{e}$ the temperatures of both species and $n_{0}$ the (assumed constant and isotropic) density of the plasma at equilibrium. The variables are $B, E$ the electromagnetic field, $v_{e}, v_{i}$ the velocities of the electrons and of the ions, and $n_{e}$ and $n_{i}$ the density fluctuations from the equilibrium $n_{0}$.

The nonlinear term in the Ampère equation is the current density term. The nonlinear terms in the equations of conservation of momentum are the Lorentz forces terms. 
The mass of the electrons is small compared to the mass of the ions : $m_{e} \ll m_{i}$. The Lorentz force is the same for the ions and the electrons, therefore the velocity of the ions is negligible compared to the velocity of the electrons. As a consequence, we neglect the contribution of the ions in the Ampre equation.

We restrict to longitudinal waves, that is

$$
\nabla \times B=0, \quad \nabla \times E=0, \quad \nabla \times v_{e}=0,
$$

at first order. Neglecting most of the nonlinear terms, these waves satisfy the following relation:

$$
\begin{aligned}
\partial_{t} B & =0, \\
\partial_{t} E & =4 \pi e\left(n_{0}+n_{e}\right) v_{e}, \\
\partial_{t} v_{e} & =-\frac{\gamma_{e} T_{e}}{m_{e} n_{0}} \nabla n_{e}-\frac{e}{m_{e}} E, \\
\partial_{t} n_{e}+n_{0} \nabla \cdot v_{e} & =0,
\end{aligned}
$$

Taking the time derivative of (2.28) and using (2.29), we obtain

$$
\partial_{t}^{2} v_{e}=\frac{\gamma_{e} T_{e}}{m_{e}} \nabla\left(\nabla \cdot v_{e}\right)-\frac{4 \pi e^{2}}{m_{e}}\left(n_{0}+n_{e}\right) v_{e} .
$$

That is

$$
\left(\partial_{t}^{2}-v_{T e}^{2} \Delta+\omega_{p e}^{2}\right) v_{e}=-\frac{\omega_{p e}^{2}}{n_{0}} n_{e} v_{e}
$$

where $\omega_{p e}$ is the electronic plasma pulsation

$$
\omega_{p e}=\sqrt{\frac{4 \pi e^{2} n_{0}}{m_{e}}},
$$

and $v_{T e}$ is the thermal velocity of the electrons

$$
v_{T e}=\sqrt{\frac{\gamma_{e} T_{e}}{m_{e}}} .
$$


For plasmas created by lasers, a typical value is $\omega_{p e}=10^{14} \mathrm{~s}^{-1}$. Using the time enveloppe approximation:

$$
E=e^{-i \omega_{p e} t} \tilde{E}, \quad v_{e}=e^{-i \omega_{p e} t} \tilde{v}_{e}
$$

where $\tilde{E}, \tilde{v}_{e}$ are slowly varying enveloppes, in the sense that

$$
\left|\partial_{t} \tilde{E}\right| \ll \omega_{p e}|\tilde{E}|, \quad\left|\partial_{t} \tilde{v}_{e}\right| \ll \omega_{p e}\left|v_{e}\right|
$$

(2.27) gives

$$
-i \omega_{p e} \tilde{E}=4 \pi e n_{o} \tilde{v}_{e}
$$

and the equation for the enveloppe of the field is

$$
\left(\partial_{t}^{2}-v_{T e}^{2} \Delta+\omega_{p e}^{2}\right) \tilde{E}=-\omega_{p e}^{2} \frac{n_{0}}{n_{e}} \tilde{E}
$$

Using $T$ and $L$ as typical space and time sizes, the equation becomes

$$
\left(\partial_{t}^{2}-\frac{T^{2} v_{T e}^{2}}{L^{2}} \Delta+\omega_{p e}^{2} T^{2}\right) \tilde{E}=-\omega_{p e}^{2} T^{2} \frac{n_{e}}{n_{0}} \tilde{E} .
$$

Now one sets:

$$
T \omega_{p e}=\frac{1}{\varepsilon}, \quad \frac{T v_{T e}}{L}=\frac{1}{\sqrt{\varepsilon}} .
$$

This gives

$$
\left(\partial_{t}^{2}-\frac{1}{\varepsilon} \Delta+\frac{1}{\varepsilon^{2}}\right) \tilde{E}=-\frac{1}{\varepsilon^{2}} \frac{n_{e}}{n_{0}} \tilde{E} .
$$

Now for initial data of characteristic size $\varepsilon$, we have $\tilde{E}=O(\varepsilon)$ and $n_{e} / n_{0}=$ $O\left(\varepsilon^{2}\right)$ (see [27]). This eventually gives the first equation of (1.10). The second equation in (1.10) is obtained as usual by linearizing the Euler equations for the ions (see [3] for more details, or a physics textbook as [7]).

We now show how (1.10) can be tranformed into a semilinear system of the form (1.9). Let

$$
u_{1}=\varepsilon \partial_{t} E, \quad u_{2}:=\sqrt{\varepsilon} \nabla E, \quad u_{3}:=E, \quad m_{1}:=n, \quad m_{2}:=\nabla \Delta^{-1} \partial_{t} n .
$$


For the unknown $\left(u_{1}, u_{2}, u_{3}, m_{1}, m_{2}\right)$, the system reads

$$
\left\{\begin{aligned}
& \partial_{t} u_{1}-\frac{1}{\sqrt{\varepsilon}} \nabla u_{2}+\frac{1}{\varepsilon} u_{3}= m_{1} u_{3}, \\
& \partial_{t} u_{2}-\frac{1}{\sqrt{\varepsilon}} \nabla u_{1}= 0 \\
& \partial_{t} u_{3}-\frac{1}{\varepsilon} u_{1}= 0 \\
& \partial_{t} m_{1}-\nabla \cdot m_{2}= 0 \\
& \partial_{t} m_{2}-\nabla m_{1}=\frac{2}{\sqrt{\varepsilon}} u_{3} u_{2}
\end{aligned}\right.
$$

(2.32) is a system of the form (1.9). We present in section 2.3 an abstract setting for such a transformation.

\subsection{A quasilinear, one-dimensional Klein-Gordon wave system}

Consider the system (1.12) in the form

$$
\left\{\begin{aligned}
\partial_{t}\left(\begin{array}{l}
u \\
v
\end{array}\right)+\frac{1}{\sqrt{\varepsilon}} \partial_{x}\left(\begin{array}{l}
v \\
u
\end{array}\right) & =\left(n+\frac{1}{\varepsilon}\right)\left(\begin{array}{c}
v \\
-u
\end{array}\right), \\
\partial_{t}\left(\begin{array}{l}
n \\
\varphi
\end{array}\right)+\partial_{x}\left(\begin{array}{l}
\varphi \\
n
\end{array}\right) & =\partial_{x}\left(\begin{array}{c}
0 \\
u^{2}+v^{2}
\end{array}\right) .
\end{aligned}\right.
$$

This is a one-dimensional version of the Klein-Gordon-wave system (1.10). We give a polynomial change of variable that transforms it into a semilinear system.

First perform the linear change of variable (diagonalization);

$$
U=\frac{1}{\sqrt{2}}(u+v), \quad V=\frac{1}{\sqrt{2}}(u-v), \quad N_{1}=n+\varphi, \quad N_{2}=n-\varphi
$$


Then we have

$$
\left\{\begin{aligned}
\partial_{t}\left(\begin{array}{c}
U \\
V
\end{array}\right)+\frac{1}{\sqrt{\varepsilon}} \partial_{x}\left(\begin{array}{c}
U \\
-V
\end{array}\right) & =\left(\frac{1}{2}\left(N_{1}+N_{2}\right)+\frac{1}{\varepsilon}\right)\left(\begin{array}{c}
-V \\
U
\end{array}\right), \\
\partial_{t}\left(\begin{array}{c}
N_{1} \\
N_{2}
\end{array}\right)+\partial_{x}\left(\begin{array}{c}
N_{1} \\
-N_{2}
\end{array}\right) & =\partial_{x}\left(\begin{array}{c}
U^{2}+V^{2} \\
-\left(U^{2}+V^{2}\right) .
\end{array}\right)
\end{aligned}\right.
$$

Now consider the polynomial change of variables:

$$
\tilde{N}_{1}=N_{1}+\alpha U^{2}+\beta V^{2}, \quad \tilde{N}_{2}=N_{2}+\gamma U^{2}+\delta V^{2}
$$

where $\alpha, \beta, \gamma, \delta$ are real constants to be fixed later. We have

$$
\begin{aligned}
\partial_{t} \tilde{N}_{1} & =\partial_{t} N_{1}+\alpha \partial_{t}\left(U^{2}\right)+\beta \partial_{t}\left(V^{2}\right) \\
& =-\partial_{x}\left(\tilde{N}_{1}-\alpha U^{2}-\beta V^{2}\right)+\partial_{x}\left(U^{2}+V^{2}\right) \\
& -\frac{\alpha}{\sqrt{\varepsilon}} \partial_{x}\left(U^{2}\right)+\frac{\beta}{\sqrt{\varepsilon}} \partial_{x}\left(V^{2}\right)+(\beta-\alpha)\left(n+\frac{1}{\varepsilon}\left(U^{2}+V^{2}\right)\right) .
\end{aligned}
$$

Thus choosing $\alpha$ and $\beta$ as

$$
\alpha:=\frac{\sqrt{\varepsilon}}{1-\sqrt{\varepsilon}}, \quad \beta:=\frac{-\sqrt{\varepsilon}}{1+\sqrt{\varepsilon}},
$$

the equation for $\tilde{N}_{1}$ becomes

$$
\partial_{t} \tilde{N}_{1}+\partial_{x} \tilde{N}_{1}=\frac{-2 \sqrt{\varepsilon}}{1-\varepsilon}\left(n+\frac{1}{\varepsilon}\right) U V .
$$

Similarly, if one lets

$$
\gamma:=\beta, \quad \delta:=\alpha
$$

one obtains

$$
\partial_{t} \tilde{N}_{2}-\partial_{x} \tilde{N}_{2}=\frac{2 \sqrt{\varepsilon}}{1-\varepsilon}\left(n+\frac{1}{\varepsilon}\right) U V .
$$

The equation for $(U, V)$ is

$\partial_{t}\left(\begin{array}{c}U \\ V\end{array}\right)+\partial_{x}\left(\begin{array}{c}U \\ -V\end{array}\right)=\left(\frac{1}{2}\left(\tilde{N}_{1}+\tilde{N}_{2}\right)+\frac{\varepsilon}{1-\varepsilon}\left(U^{2}+V^{2}\right)+\frac{1}{\varepsilon}\right)\left(\begin{array}{c}-V \\ U\end{array}\right)$.

The system (2.35)-(2.36)-(2.37) has the form (1.7) with $d(\varepsilon)=1$ and $c(\varepsilon)=$ $\sqrt{\varepsilon}$. 


\subsection{Nonlinear conjugation with quasilinear systems}

We give in this section an abtract setting for the change of variables described above. Consider quasilinear hyperbolic systems of the form (1.23). The physical relevance of such systems is given in the previous section. We prove in this section Proposition 1.12. The validity of Assumption 1.11 is discussed in section 2.4.2. We consider the regime $c(\varepsilon)=\sqrt{\varepsilon}$ below; in this paragraph, any $c(\varepsilon) \neq 1$ is appropriate.

With the above notations for the spectral decomposition of $A$ and $B$, one has for all $\eta$ :

$$
\mathrm{Id}=\sum_{k} P_{k}(\eta)=\sum_{l} Q_{l}(\eta)
$$

The eigenvalues $\lambda_{k}$ and $\mu_{l}$ are continuous, and they have directional derivatives in every direction at every point $[17,26]$.

Proof of Proposition 1.12. If $(u, m)$ is a solution of (1.23), then for any bilinear map $S^{\varepsilon}$ and for any change of variables of the form (1.24),

$$
\left(\partial_{t}+B\left(\partial_{x}\right)\right) \tilde{m}=-\partial_{t} S^{\varepsilon}(\tilde{u}, \tilde{u})-B\left(\partial_{x}\right) S^{\varepsilon}(\tilde{u}, \tilde{u})+\partial_{x} g(u, u)
$$

One wants to find $S^{\varepsilon}$ such that the right hand side of the above equation involves no derivatives of $\tilde{u}$. With the form of the operators involved in (1.23), it is natural to look for $S^{\varepsilon}$ in the form of a constant coefficient bilinear pseudodifferential operator:

$$
S^{\varepsilon}(v, v):=\int_{\eta, \eta^{\prime} \in \mathbb{R}^{d}} \exp \left(i x \cdot\left(\eta+\eta^{\prime}\right)\right) S_{\eta, \eta^{\prime}}^{\varepsilon}\left(\hat{v}(\eta), \hat{v}\left(\eta^{\prime}\right)\right) d \eta d \eta^{\prime} .
$$

Then one has

$$
\begin{aligned}
\partial_{t} S^{\varepsilon}(\tilde{u}, \tilde{u}) & =S^{\varepsilon}\left(\partial_{t} \tilde{u}, \tilde{u}\right)+S^{\varepsilon}\left(\tilde{u}, \partial_{t} \tilde{u}\right) \\
& =-S^{\varepsilon}\left(A\left(\partial_{x}\right) \tilde{u}, \tilde{u}\right)-S^{\varepsilon}\left(\tilde{u}, A\left(\partial_{x}\right) \tilde{u}\right)+(\operatorname{order} 0)(\tilde{u}, \tilde{m})
\end{aligned}
$$


and

$$
\begin{aligned}
\left(\partial_{t}+B\left(\partial_{x}\right)\right) \tilde{m} & -\frac{1}{c(\varepsilon)} S^{\varepsilon}\left(A\left(\partial_{x}\right) \tilde{u}, \tilde{u}\right)-\frac{1}{c(\varepsilon)} S^{\varepsilon}\left(\tilde{u}, A\left(\partial_{x}\right) \tilde{u}\right) \\
& +B\left(\partial_{x}\right) S^{\varepsilon}(\tilde{u}, \tilde{u})-\partial_{x} g(\tilde{u}, \tilde{u})+(\text { order } 0)(\tilde{u}, \tilde{m})=0 .
\end{aligned}
$$

The homological equation is

$$
c(\varepsilon) B\left(\partial_{x}\right) S^{\varepsilon}(\tilde{u}, \tilde{u})-S^{\varepsilon}\left(A\left(\partial_{x}\right) \tilde{u}, \tilde{u}\right)-S^{\varepsilon}\left(\tilde{u}, A\left(\partial_{x}\right) \tilde{u}\right)=c(\varepsilon) \partial_{x} g(\tilde{u}, \tilde{u}) .
$$

In Fourier modes:

$$
\begin{aligned}
c(\varepsilon) B\left(i\left(\eta+\eta^{\prime}\right)\right) S_{\eta, \eta^{\prime}}^{\varepsilon}\left(\hat{u}(\eta), \hat{u}\left(\eta^{\prime}\right)\right) & -S_{\eta, \eta^{\prime}}^{\varepsilon}\left(A(i \eta) \hat{u}(\eta), \hat{u}\left(\eta^{\prime}\right)\right)-S_{\eta, \eta^{\prime}}^{\varepsilon}\left(\hat{u}(\eta), A\left(i \eta^{\prime}\right) \hat{u}\left(\eta^{\prime}\right)\right) \\
& =i\left(\eta+\eta^{\prime}\right) g\left(\left(\hat{u}(\eta), \hat{u}\left(\eta^{\prime}\right)\right) .\right.
\end{aligned}
$$

When $\eta+\eta^{\prime}=0$, one sets $S^{\varepsilon}\left(\eta, \eta^{\prime}\right)=0$. When $\eta+\eta^{\prime} \neq 0$, a sufficient condition is to have, for all $\eta, \eta^{\prime} \in \mathbb{R}^{d}$, and for all vectors $u, u^{\prime}$ in $\mathbb{C}^{N}$,

$$
g\left(u, u^{\prime}\right)=c(\varepsilon) B\left(\eta+\eta^{\prime}\right) S_{\eta, \eta^{\prime}}^{\varepsilon}\left(u, u^{\prime}\right)-S_{\eta, \eta^{\prime}}^{\varepsilon}\left(A(\eta) u, u^{\prime}\right)-S_{\eta, \eta^{\prime}}^{\varepsilon}\left(u, A\left(\eta^{\prime}\right) u^{\prime}\right) .
$$

With the spectral decompositions of $A$ and $B$, this gives

$$
\begin{aligned}
c(\varepsilon)\left(\eta+\eta^{\prime}\right) Q_{l}\left(\eta+\eta^{\prime}\right) g\left(P_{k}(\eta) u, P_{k^{\prime}}\left(\eta^{\prime}\right) u^{\prime}\right)= & \left(c(\varepsilon) \mu_{l}\left(\eta+\eta^{\prime}\right)-\lambda_{k}(\eta)-\lambda_{k^{\prime}}\left(\eta^{\prime}\right)\right) \\
& \times Q_{l}\left(\eta+\eta^{\prime}\right) S_{\eta, \eta^{\prime}}^{\varepsilon}\left(P_{k}(\eta) u, P_{k^{\prime}}\left(\eta^{\prime}\right) u^{\prime}\right) .
\end{aligned}
$$

Then with Assumption 1.11, setting for all $u, u^{\prime}, k, k^{\prime}, l, \eta, \eta^{\prime}$,

$$
\begin{gathered}
Q_{l}\left(\eta+\eta^{\prime}\right) S_{\eta, \eta^{\prime}}^{\varepsilon}\left(P_{k}(\eta) u, P_{k^{\prime}}\left(\eta^{\prime}\right) u^{\prime}\right) \\
=\frac{c(\varepsilon)\left(\eta+\eta^{\prime}\right)}{c(\varepsilon) \mu_{l}\left(\eta+\eta^{\prime}\right)-\lambda_{k}(\eta)-\lambda_{k^{\prime}}\left(\eta^{\prime}\right)} Q_{l}\left(\eta+\eta^{\prime}\right) g\left(P_{k}(\eta) u, P_{k^{\prime}}\left(\eta^{\prime}\right) u^{\prime}\right),
\end{gathered}
$$

one defines a bounded family of bilinear maps $\left\{S^{\varepsilon}\right\}_{\varepsilon \in] 0,1[}$ (because the growth of $\mu_{l}$ and $\lambda_{k}$ is at most linear). The dominant term in the 0 order operator in the equation for $\tilde{m}$ has coefficient $c(\varepsilon) / \varepsilon$. This ends the proof of the proposition.

Remark 2.1 This is a high-frequency analysis, that is only the principal symbols of $L^{\varepsilon}$ and $M^{\varepsilon}$ are in play in the construction of $S^{\varepsilon}$, but it is the dispersion term $L_{0}$ that gives the coefficient $c(\varepsilon) / \varepsilon$. 


\subsection{Verification of the assumptions on the model sys- tems}

\subsubsection{Verification of Assumptions 1.4, 1.5 and 1.7}

Verification on (1.12). Consider (1.12) in the form (2.34). It has the form (1.9) with

$$
A=B:=\left(\begin{array}{cc}
1 & 0 \\
0 & -1
\end{array}\right), \quad L_{0}:=\left(\begin{array}{cc}
0 & 1 \\
-1 & 0
\end{array}\right)
$$

and

$$
g\left(u, u^{\prime}\right):=\frac{1}{2}\left(\begin{array}{c}
u_{1} u_{2}^{\prime}+u_{1}^{\prime} u_{2} \\
-\left(u_{1} u_{2}^{\prime}+u_{1}^{\prime} u_{2}\right)
\end{array}\right) .
$$

The eigenvalues of $A(\eta) / \sqrt{\varepsilon}+L_{0} / i$ are $\pm \lambda^{\varepsilon}(\eta)= \pm \sqrt{1+\eta^{2} / \varepsilon}$. The associated eigenvectors are

$$
e_{ \pm}^{\varepsilon}(\eta):=\left(\begin{array}{c}
1 \\
i\left( \pm \lambda^{\varepsilon}(\eta)-\frac{\eta}{\sqrt{\varepsilon}}\right)
\end{array}\right) .
$$

The eigenvalues of $B(\eta)$ are $\pm \eta$. The associated eigenvectors are $f_{+}:=(1,0)$ and $f_{-}:=(0,1)$. The characteristic variety of this system is the union of two Klein-Gordon branches and two acoustic waves branches. Compare with the characteristic variety of the Euler-Maxwell system as described in [27]. One has

$$
g\left(e_{+}(0), e_{-}(0)\right)=0 .
$$

This is Assumption 1.4. The points $\beta=(1,0)$ and $-\beta=(-1,0)$ are respectively a local minimum and a local maximum of the characteristic variety. This is Assumption 1.5. The interaction coefficient is

$$
c(\tilde{\eta}):=<f_{+}, g\left(e_{-}(0), e_{+}^{\varepsilon}(\tilde{\eta})\right)>=i\left(\sqrt{1+\frac{|\tilde{\eta}|^{2}}{\varepsilon}}-\frac{|\tilde{\eta}|}{\sqrt{\varepsilon}}-1\right) .
$$

Let $\xi^{\prime}:=(|\tilde{\eta}|, \tilde{\eta})$, and $\xi:=\left(\lambda^{\varepsilon}(\tilde{\eta}), \tilde{\eta}\right)$. The condition $\left|\tau^{\prime}-(\tau-\omega)\right| \leq \sqrt{\varepsilon}$ is 
explicitly

$$
|| \tilde{\eta}\left|+1-\sqrt{1+\frac{|\tilde{\eta}|^{2}}{\varepsilon}}\right| \leq \sqrt{\varepsilon} .
$$

We will use the uniform bounds: for all $x$,

$$
1+\frac{1}{2}|x|^{2}-\frac{1}{8}|x|^{4} \leq \sqrt{1+|x|^{2}} \leq 1+\left.\left|\frac{1}{2}\right| x\right|^{2} .
$$

Let $\tilde{\eta}=\sqrt{\varepsilon} \eta$, and

$$
\psi(\eta):=\sqrt{\varepsilon}|\eta|+1-\sqrt{1+|\eta|^{2}} .
$$

The interaction coefficient is

$$
c(\eta)=\sqrt{1+|\eta|^{2}}-|\eta|-1 .
$$

The bound (2.39) is equivalent to $|\psi| \leq \sqrt{\varepsilon}$. Hence we have

$$
\frac{1}{2}|\eta|^{2}-\frac{1}{8}|\eta|^{4}-\sqrt{\varepsilon}|\eta| \leq \sqrt{\varepsilon} .
$$

This implies the existence of $\varepsilon_{1}>0$ and $c_{1}>\sqrt{2}$ such that for $0<\varepsilon<\varepsilon_{1}$, if (2.39) is satisfied, then $|\eta| \leq c_{1} \varepsilon^{1 / 4}$.

When $|\psi(\eta)| \leq \varepsilon$, we have

$$
\frac{1}{2}|\eta|^{2}-\frac{1}{8}|\eta|^{4}-\sqrt{\varepsilon}|\eta| \leq \varepsilon .
$$

Thus there exists $0<\varepsilon_{2} \leq \varepsilon_{1}$ and $c_{2}>0,2<c_{2}<3$, such that for all $0<\varepsilon<\varepsilon_{2},|\eta| \leq c_{2} \sqrt{\varepsilon}$. Then the interaction coefficient can be directly bounded as

$$
|c(\eta)| \leq|\eta|\left(1+\frac{1}{2}|\eta|\right) \leq c_{2} \sqrt{\varepsilon}\left(1+c_{2} \sqrt{\varepsilon}\right) .
$$

When $\varepsilon \leq|\psi(\eta)| \leq \sqrt{\varepsilon}$, we have

$$
\varepsilon \leq \frac{1}{2}|\eta|^{2}-\sqrt{\varepsilon}|\eta|,
$$

hence $|\eta| \geq(1+\sqrt{3}) \sqrt{\varepsilon}$. Thus for $\varepsilon_{0}$ small enough and $0<\varepsilon<\varepsilon_{0}$,

$$
\begin{aligned}
\left|\frac{\varepsilon c(\eta)}{\psi(\eta)}\right| & \leq \varepsilon \frac{|\eta|\left(1+\frac{1}{2}|\eta|\right)}{\frac{1}{2}|\eta|^{2}-\sqrt{\varepsilon}|\eta|-\frac{1}{8}|\eta|^{4}} \\
& \leq c_{3} \sqrt{\varepsilon},
\end{aligned}
$$


with

$$
c_{3}:=\frac{1+c_{1} \varepsilon_{0}^{1 / 4}}{\frac{1}{2}(1+\sqrt{3})-1-\frac{1}{8} c_{1}^{2} \varepsilon_{0}^{1 / 2}} .
$$

One chooses $0<\varepsilon_{0} \leq \min \left(\varepsilon_{1}, \varepsilon_{2}\right)$ small enough such that $c_{3}>0$. Finally, with

$$
C:=\sup \left(c_{2}\left(1+c_{2} \sqrt{\varepsilon}_{0}\right), c_{3}\right),
$$

Assumption 1.7 is satisfied.

Verification on (1.10). Consider (1.10) in the form (2.32). It has the form (1.9) with

$$
A(\eta):=\left(\begin{array}{ccc}
0 & -\eta \cdot & 0 \\
-\eta & 0 & 0 \\
0 & 0 & 0
\end{array}\right), \quad L_{0}:=\left(\begin{array}{ccc}
0 & 0 & 1 \\
0 & 0 & 0 \\
-1 & 0 & 0
\end{array}\right), \quad B(\eta):=\left(\begin{array}{cc}
0 & \eta \cdot \\
\eta & 0
\end{array}\right)
$$

and

$$
g(u, u):=\left(\begin{array}{c}
0 \\
u_{2} u_{3}^{\prime}+u_{2}^{\prime} u_{3}
\end{array}\right) .
$$

For $\eta \neq 0$, the eigenvectors of $A(\eta) / \sqrt{\varepsilon}+L_{0} / i$ associated with the eigenvalues $\pm \lambda= \pm \sqrt{1+|\eta|^{2} / \varepsilon}$ are

$$
e_{ \pm}(\eta):=\left(\begin{array}{c}
1 \\
-\frac{\eta}{ \pm \lambda \sqrt{\varepsilon}} \\
\frac{i}{ \pm \lambda}
\end{array}\right)
$$

This gives

$$
g\left(e_{+}(0), e_{-}(0)\right)=g\left(e_{-}(0), e_{+}(0)\right)=0 .
$$

Again, $\beta$ and $-\beta$ are local extrema of the characteristic variety and Assumptions 1.4 and 1.5 are satisfied. The interaction coefficient is

$$
<f_{+}(\eta), g\left(e_{-}(0), e_{+}\left(\eta^{\prime}\right)\right)>=\frac{\eta}{|\eta|} \cdot \frac{\eta^{\prime}}{\sqrt{\varepsilon}} \frac{i}{\sqrt{1+\left|\frac{\eta^{\prime}}{\sqrt{\varepsilon}}\right|^{2}}} .
$$

One checks that Assumption 1.7 is satisfied; the computations are the same as in the previous example. 


\subsubsection{Verification of Assumption 1.11}

We now discuss the validity of Assumption 1.11 on (1.12) and (1.13) (there is nothing to check for (1.10) as it is actually semilinear). In these examples, all the resonances are regular (see [10]), so that in order to check Assumption 1.7 , one only needs to check that the interaction coefficients vanish at the resonances.

Verification on (1.12). Consider (1.12) in the form (2.33). It has the form (1.23) with

$$
A=B=\left(\begin{array}{ll}
0 & 1 \\
1 & 0
\end{array}\right), \quad g\left(u, u^{\prime}\right)=\left(\begin{array}{c}
0 \\
u_{1} u_{1}^{\prime}+u_{2} u_{2}^{\prime}
\end{array}\right) .
$$

The eigenvalues of $A(\eta)$ and $B(\eta)$ are $\pm|\eta|$, the associated eigenvectors are

$$
e_{ \pm}(\eta)=\frac{1}{\sqrt{2}}\left(\begin{array}{c}
1 \\
\pm \frac{\eta}{|\eta|}
\end{array}\right)
$$

and the eigenprojectors are $P_{ \pm}(\eta)=Q_{ \pm}(\eta)=e_{ \pm}(\eta) \otimes e_{ \pm}(\eta)$. The resonances are given by the four equations

$$
\pm c(\varepsilon)\left|\eta \pm \eta^{\prime}\right|=|\eta|-\left|\eta^{\prime}\right|
$$

For symmetry reasons, it suffices to consider the resonances for the positive branch of the acoustic wave equation, that is to restrict to the resonance equation

$$
c(\varepsilon)\left|\eta \pm \eta^{\prime}\right|=|\eta|-\left|\eta^{\prime}\right|
$$

For a given $\eta>0$, the solutions are

$$
\eta^{\prime}= \pm \frac{1-\sqrt{\varepsilon}}{1+\sqrt{\varepsilon}} \eta, \quad \text { and } \quad \eta^{\prime}=-\eta .
$$

Then for $\eta^{\prime}= \pm \frac{1-\sqrt{\varepsilon}}{1+\sqrt{\varepsilon}} \eta$, one has

$$
Q_{+}\left(\eta+\eta^{\prime}\right) g\left(P_{+}(\eta) u, P_{ \pm}\left(\eta^{\prime}\right) u^{\prime}\right)=<u, e_{+}(\eta)><u^{\prime}, e_{ \pm}\left(\eta^{\prime}\right)>\left(1 \mp \frac{\eta}{|\eta|} \frac{\eta^{\prime}}{\left|\eta^{\prime}\right|}\right)
$$


where $<\cdot, \cdot>$ denotes the scalar product in $\mathbb{R}^{2}$. The interaction coefficient is

$$
c_{ \pm}\left(\eta, \eta^{\prime}\right)=1 \mp \frac{\eta}{|\eta|} \frac{\eta^{\prime}}{\left|\eta^{\prime}\right|}
$$

When $\eta^{\prime}=(1-\sqrt{\varepsilon})(1+\sqrt{\varepsilon})^{-1} \eta, \eta$ and $\eta^{\prime}$ have the same sign, and $c_{+}\left(\eta, \eta^{\prime}\right)=0$. When $\eta^{\prime}=-(1-\sqrt{\varepsilon})(1+\sqrt{\varepsilon})^{-1} \eta, \eta$ and $\eta^{\prime}$ have different signs, and $c_{-}\left(\eta, \eta^{\prime}\right)=$ 0 . Thus the interaction coefficient vanishes at the resonances and Assumption 1.11 is satisfied.

Verification on (1.13). Consider (1.13). It has the form (1.23) with

$$
A(\eta)=A\left(\eta_{1}, \eta_{2}\right)=\left(\begin{array}{cc}
-\eta_{1} & \eta_{2} \\
\eta_{2} & \eta_{1}
\end{array}\right), \quad B(\eta)=\left(\begin{array}{ccc}
0 & \eta_{1} & \eta_{2} \\
\eta_{1} & 0 & 0 \\
\eta_{2} & 0 & 0
\end{array}\right)
$$

and

$$
g\left(u, u^{\prime}\right):=\left(\begin{array}{c}
0 \\
g_{1}\left(u, u^{\prime}\right) \\
g_{1}\left(u, u^{\prime}\right)
\end{array}\right)
$$

with $g_{1}\left(u, u^{\prime}\right):=u_{1} u_{1}^{\prime}+u_{2} u_{2}^{\prime}$. The system is

$$
\left\{\begin{aligned}
\left(\partial_{t}+\frac{1}{c(\varepsilon)} A\left(\partial_{x}, \partial_{y}\right)+L_{0} / \varepsilon\right) u^{\varepsilon} & =f\left(u^{\varepsilon}, m^{\varepsilon}\right), \\
\left(\partial_{t}+B\left(\partial_{x}, \partial_{y}\right)\right) m^{\varepsilon} & =\left(\begin{array}{c}
0 \\
\partial_{x} g_{1}\left(u^{\varepsilon}, u^{\varepsilon}\right) \\
\partial_{y} g_{1}\left(u^{\varepsilon}, u^{\varepsilon}\right)
\end{array}\right)
\end{aligned}\right.
$$

The eigenvalues of $A(\eta)$ (resp. $B(\eta))$ are $\pm|\eta|($ resp. $0, \pm|\eta|)$, where $|\cdot|$ is the Euclidean norm in $\mathbb{R}^{2}$. One has $A(\eta)= \pm|\eta| e_{ \pm}(\eta)$, with

$$
e_{ \pm}(\eta)=\left(\begin{array}{c}
1 \\
\frac{ \pm|\eta|+\eta_{1}}{\eta_{2}}
\end{array}\right) .
$$


It is not restrictive to suppose $\eta_{2} \neq 0$ in the following computations. One has $B(\eta) f_{ \pm}(\eta)= \pm|\eta| f_{ \pm}(\eta)$, with

$$
f_{ \pm}(\eta)=\left(\begin{array}{c}
1 \\
\pm \frac{\eta_{1}}{|\eta|} \\
\pm \frac{\eta_{2}}{|\eta|}
\end{array}\right)
$$

and $B(\eta) f_{0}(\eta)=0$, with

$$
f_{0}(\eta)=\left(\begin{array}{c}
0 \\
1 \\
\frac{-\eta_{1}}{\eta_{2}}
\end{array}\right) .
$$

The resonance equations are

$$
\pm c(\varepsilon)\left|\eta \pm \eta^{\prime}\right|=|\eta|-\left|\eta^{\prime}\right|
$$

Again, it suffices to consider the positive branch of the acoustic wave equation, that is to restrict to

$$
c(\varepsilon)\left|\eta \pm \eta^{\prime}\right|=|\eta|-\left|\eta^{\prime}\right| .
$$

The resonances with the + sign are deduced from the resonances with the - sign by changing $\theta$ into $\theta+\pi$ (where $\eta=|\eta| e^{i \theta}$ ) or $\theta^{\prime}$ into $\theta^{\prime}+\pi$ (where $\eta=|\eta| e^{i \theta}$ ). If $|\eta| \neq\left|\eta^{\prime}\right|$, then (2.40) with the - sign is equivalent to

$$
c(\varepsilon)^{2}\left(|\eta|^{2}+\left|\eta^{\prime}\right|^{2}-2|\eta|\left|\eta^{\prime}\right| \cos \left(\theta-\theta^{\prime}\right)\right)=|\eta|^{2}+\left|\eta^{\prime}\right|^{2}-2|\eta \|| \eta^{\prime} \mid
$$

that is

$$
2 \frac{1-c(\varepsilon)^{2} \cos \left(\theta-\theta^{\prime}\right)}{1-c(\varepsilon)^{2}}=\frac{|\eta|}{\eta^{\prime} \mid}+\frac{\left|\eta^{\prime}\right|}{\eta \mid} .
$$

Resonances occur for all values of $\eta \in] 0,+\infty[$. Therefore the right hand side can take any value in $[2,+\infty[$, and the above equation gives a void condition for the angular variables: $\cos \left(\theta-\theta^{\prime}\right) \leq 1$. The interaction coefficient is $c\left(\theta, \theta^{\prime}\right):=\frac{1}{\left|\eta+\eta^{\prime}\right|^{2}}<f_{+}\left(\eta+\eta^{\prime}\right), g\left(e_{+}(\eta), e_{+}\left(\eta^{\prime}\right)>=\left(1+\frac{1+\cos \theta}{\sin \theta} \frac{1+\cos \theta^{\prime}}{\sin \theta^{\prime}}\right) \neq 0\right.$ 
at the resonance, since the angular variables $\theta, \theta^{\prime}$ can take any value in $[0,2 \pi[$.

The other resonances are given by the relation $|\eta|=\left|\eta^{\prime}\right|$. One has

$$
<f_{0}\left(\eta+\eta^{\prime}\right), g\left(e_{+}(\eta), e_{ \pm}\left(\eta^{\prime}\right)>=0\right.
$$

These resonances are transparent.

We now see how $g$ should be modified in order to satisfy Assumption 1.11. We add to $g$ a pseudodifferential term :

$$
\tilde{g}\left(u, u^{\prime}\right):=\int_{\eta, \eta^{\prime} \in \mathbb{R}^{d}} \exp \left(i x \cdot\left(\eta+\eta^{\prime}\right)\right) i\left(\eta+\eta^{\prime}\right) \tilde{g}\left(\theta, \theta^{\prime}\right)\left(\hat{u}(\eta), \hat{u}\left(\eta^{\prime}\right)\right) d \eta d \eta^{\prime} .
$$

One has again

$$
<f_{0}\left(\eta+\eta^{\prime}\right), \tilde{g}\left(e_{+}(\eta), e_{ \pm}\left(\eta^{\prime}\right)>=0,\right.
$$

hence a sufficient condition to impose on $\tilde{g}$ in order that $g+\tilde{g}$ satisfies Assumption 1.11 is

$$
<f_{+}\left(\eta+\eta^{\prime}\right), \tilde{g}\left(e_{+}(\eta), e_{+}\left(\eta^{\prime}\right)>+\left|\eta+\eta^{\prime}\right|^{2} c\left(\theta, \theta^{\prime}\right)=0,\right.
$$

for all $\eta, \eta^{\prime}$ such that $c(\varepsilon)\left|\eta+\eta^{\prime}\right|=|\eta|-\left|\eta^{\prime}\right|$. One sets

$$
\tilde{g}\left(\theta, \theta^{\prime}\right)\left(u, u^{\prime}\right)=\underline{c}\left(\theta, \theta^{\prime}\right)\left(u_{1} u_{2}^{\prime}+u_{1}^{\prime} u_{2}\right) .
$$

The condition becomes

$$
\underline{c}\left(\theta, \theta^{\prime}\right)\left(\frac{1+\cos \theta}{\sin \theta}+\frac{1+\cos \theta^{\prime}}{\sin \theta^{\prime}}\right)+c\left(\theta, \theta^{\prime}\right)=0 .
$$

When

$$
\frac{1+\cos \theta}{\sin \theta}+\frac{1+\cos \theta^{\prime}}{\sin \theta^{\prime}}=0
$$

one has $c\left(\theta, \theta^{\prime}\right)=0$ hence one sets $\underline{c}\left(\theta, \theta^{\prime}\right)=0$. Otherwise, one sets

$$
\underline{c}\left(\theta, \theta^{\prime}\right)=-\left(\frac{1+\cos \theta}{\sin \theta}+\frac{1+\cos \theta^{\prime}}{\sin \theta^{\prime}}\right)^{-1} c\left(\theta, \theta^{\prime}\right) .
$$

It follows that $A, B$ and $g+\tilde{g}$ satisfies Assumption 1.11. 


\section{The approximate WKB solution}

With the notations of the introduction, we construct in this section approximate solutions to the Cauchy problem for the system

$$
\left\{\begin{aligned}
L^{\varepsilon}\left(\partial_{t}, \partial_{x}\right) u^{\varepsilon} & =f\left(u^{\varepsilon}, m^{\varepsilon}\right), \\
M^{\varepsilon}\left(\partial_{t}, \partial_{x}\right) m^{\varepsilon} & =\frac{1}{\sqrt{\varepsilon}} g\left(u^{\varepsilon}, u^{\varepsilon}\right),
\end{aligned}\right.
$$

where $f$ and $g$ are bilinear. The initial datum is $U^{\varepsilon}(0)=\left(u^{\varepsilon}(0), m^{\varepsilon}(0)\right)=$ $\left.\left(u^{0}(x), 0\right) \in H^{\infty}\left(\mathbb{R}_{x}^{d}\right)\right)$. In view of Proposition 1.14, we want to construct ap-

proximate solutions at order 1 and defined over a time interval $\left[0, t^{*}[\right.$, with $t^{*}>0$ independent of $\varepsilon$.

We make Assumptions 1.4 and 1.5. Assumption 1.4 is a weak transparency assumption, as in $[10,4,6,27]$. It implies that the pertinent regime is given by solutions of amplitude $O(1)$ (oscillations fortes in the sense of Cheverry, Guès and Métivier [2]). Because of Assumption 1.4, solutions with amplitude $O(\sqrt{\varepsilon})$ (the weakly nonlinear regime) would indeed lead to a linear limit system.

One denotes by $\mathcal{R}$ the set of all integer $p \in \mathbb{Z}$, such that $p \beta$ is characteristic $\left(p \beta \in\right.$ Char $\left.L^{\varepsilon}\right) . \mathcal{R}$ is a finite set. For all $p \in \mathcal{R}, P(p \beta)$ is the orthogonal projector over the kernel of $\left(-p \omega+L_{0} / i\right)$.

To suppose that the eigenvalues of $A(k)+L_{0} / i$ depend on $k$ only via $|k|$ implies in particular that Char $L^{\varepsilon}$ is smooth, but several branches of Char $L^{\varepsilon}$ may intersect at $p \beta$. For example, the point $\left(\omega_{p}, 0\right)$ of the characteristic variety of the Euler-Maxwell system [27] is regular and two Klein-Gordon branches intersect at this point. As in [26], for a given $p \in \mathcal{R}$, let us consider the eigenvalues $\omega_{1}, \ldots, \omega_{s}$ of $A(k)+L_{0} / i$ that satisfy $\omega_{j}(0)=p_{0} \omega$. The corresponding (holomorphic, see Butler's theorem $[16,26]$ ) eigenprojectors are $\pi_{1}, \ldots, \pi_{s}$. Assumption 1.5 is related to the well-posedness of the profiles equations, see remark 3.1 below. 
Ansatz. We look for approximate solutions of system (3.41) in the form

$$
\begin{aligned}
U^{\varepsilon}(t, x) & =\left(u^{\varepsilon}, m^{\varepsilon}\right)(t, x)=\left[\mathbf{U}^{\varepsilon}(t, x, \theta)\right]_{\theta=\omega t / \varepsilon} \\
& =\left[\sum_{n \geq 0} \varepsilon^{n / 2} \mathbf{U}_{n}(t, x, \theta)\right]_{\theta=\omega t / \varepsilon} .
\end{aligned}
$$

This is a three-scale geometric optics approximation. $\beta=(\omega, 0)$ is a fixed characteristic phase satisfying

$$
\operatorname{det}\left(-\omega+\frac{L_{0}}{i}\right)=0
$$

The notation $u_{p}$ denotes the $p$ th Fourier coefficient of a profile $\mathbf{u}$. We use the notations $L^{\varepsilon}(\beta), M(\beta)$ introduced in (1.16). As $\beta=(\omega, 0), L^{\varepsilon}(p \beta)$ does actually not depend on $\varepsilon$, for all $p \in \mathbb{Z}$, hence we will denote this matrix by $L(p \beta)$. The equality $L(p \beta) a=b$ in $\mathbb{C}^{N}$ is equivalent to

$$
P(p \beta) b=0 \text { and }(1-P(p \beta)) a=L(p \beta)^{-1} b,
$$

where $L(p \beta)^{-1}$ is the partial inverse of $L(p \beta)$, naturally defined on the orthogonal sum ker $L(p \beta) \oplus \operatorname{ran} L(p \beta)$. One defines the operator acting on profiles:

$$
\begin{gathered}
\mathbb{L}(\beta) \mathbf{u}:=\sum_{p} e^{i p \theta} L(p \beta) u_{p}, \quad \mathbb{L}(\beta)^{-1} \mathbf{u}:=\sum_{p} e^{i p \theta} L(p \beta)^{-1} u_{p}, \\
\mathbb{P}(\beta) \mathbf{u}:=\sum_{p} e^{i p \theta} P(p \beta) u_{p}
\end{gathered}
$$

\subsection{WKB expansion}

Terms of order $O(1 / \varepsilon)$ :

$$
\left\{\begin{array}{l}
L(p \beta) u_{0, p}=0, \quad p \in \mathbb{Z}, \\
\omega \partial_{\theta} \mathbf{m}_{0}=0 .
\end{array}\right.
$$


The eikonal equation is $\operatorname{det}\left(\omega+L_{0} / i\right)=0$. The first equation gives the polarization condition:

$$
P(p \beta) u_{0, p}=u_{0, p}, p \in \mathcal{R}, \quad u_{0, p}=0, p \notin \mathcal{R} .
$$

The polarization condition for the second variable is $\mathbf{m}_{0}=m_{0,0}$.

\section{Terms of order $O(1 / \sqrt{\varepsilon})$ :}

$$
\begin{array}{r}
L(p \beta) u_{1, p}+A\left(\partial_{x}\right) u_{0, p}=0, \quad p \in \mathbb{Z} \\
\omega \partial_{\theta} \mathbf{m}_{1}=g\left(\mathbf{u}_{0}, \mathbf{u}_{0}\right)
\end{array}
$$

This implies

$$
\begin{array}{r}
P(p \beta) A\left(\partial_{x}\right) P(p \beta) u_{0, p}=0, \quad p \in \mathcal{R} \\
g\left(\mathbf{u}_{0}, \mathbf{u}_{0}\right)_{p}=0 .
\end{array}
$$

Equation (3.44) is the weak transparency equality of Assumption 1.4, which appears as a necessary condition for the construction of solutions of amplitude $O(1)$. For $p \in \mathcal{R}$, the algebraic lemma at first order at the critical point $p \beta$ implies (see [17], Proposition 2, or [26], Proposition 2.8):

$$
P(p \beta) A(\xi) P(p \beta)=\sum_{i=1}^{s} \omega_{j}^{\prime}(0) \cdot \xi \pi_{j}(0)=0,
$$

as with Assumption 1.5 one has $\omega_{j}^{\prime}(0)=0$ for all $j$. The nonpolarized part of the first corrector is:

$$
\left\{\begin{array}{l}
(1-\mathbb{P}(\beta))) \mathbf{u}_{1}=-\mathbb{L}(i \beta)^{-1} A\left(\partial_{x}\right) \mathbf{u}_{0} \\
m_{1, p}=\frac{1}{i p \omega} g\left(\mathbf{u}_{0}, \mathbf{u}_{0}\right)_{p}, \quad p \neq 0
\end{array}\right.
$$

Terms of order $O(1)$ :

$$
\left\{\begin{array}{l}
L(p \beta) u_{2, p}+A\left(\partial_{x}\right) u_{1, p}+\partial_{t} u_{0, p}=f\left(\mathbf{u}_{0}, \mathbf{m}_{0}\right)_{p}, \quad p \in \mathbb{Z} \\
\omega \partial_{\theta} \mathbf{m}_{2}+\left(\partial_{t}+M\left(\partial_{x}\right)\right) \mathbf{m}_{0}=g\left(\mathbf{u}_{0}, \mathbf{u}_{1}\right)+g\left(\mathbf{u}_{1}, \mathbf{u}_{0}\right)
\end{array}\right.
$$


This implies for $p \in \mathcal{R}$,

$$
\begin{gathered}
\left(\partial_{t}+P(p \beta) A\left(\partial_{x}\right) L(i p \beta)^{-1} A\left(\partial_{x}\right)\right) P(p \beta) u_{0, p} \\
=P(p \beta) f\left(P(p \beta) u_{0, p}, m_{0,0}\right), \quad p \in \mathcal{R} \\
\left(\partial_{t}+M\left(\partial_{x}\right)\right) m_{0,0}=g\left(\mathbf{u}_{0}, L(i \beta)^{-1} A\left(\partial_{x}\right) \mathbf{u}_{0}\right)_{0}+g\left(L(i \beta)^{-1} A\left(\partial_{x}\right) \mathbf{u}_{0}, \mathbf{u}_{0}\right)_{0}
\end{gathered}
$$

The system (3.46)-(3.47) is the announced Zakharov system. The algebraic lemma at second order at the critical point $p \beta$ (see [26], Propositions 2.6 and 2.8) reads

$$
P(p \beta) A(\xi) L(p \beta)^{-1} A(\xi) P(p \beta)=\sum_{j=1}^{s}\left(\omega_{j}^{\prime}(0) \cdot \xi\right) \pi_{j}^{\prime}(0) \xi+\omega_{j}^{\prime \prime}(0)(\xi, \xi) \pi_{j}(0),
$$

and with Assumption 1.5, one has $\omega_{j}^{\prime}(0)=0$ and the real numbers $\omega_{j}^{\prime \prime}(0)$ all have the same sign. This means that the symbol of

$$
\omega^{\prime \prime}(p \beta)\left(\partial_{x}, \partial_{x}\right) P(p \beta)=P(p \beta) A\left(\partial_{x}\right) L(p \beta)^{-1} A\left(\partial_{x}\right) P(p \beta)
$$

in a basis of $\operatorname{Ran} P(p \beta)=\oplus_{j=1}^{s} \operatorname{Ran} \pi_{j}(0)$ is a diagonal matrix with entries $\omega_{j}^{\prime \prime}(0)$. Hence it is an elliptic operator and the existence and uniqueness theorem of Ozawa and Tsutsumi [20] applies to (3.46)-(3.47). The nonpolarized part of the second corrector is:

$$
\left\{\begin{array}{l}
(1-\mathbb{P}(\beta)) \mathbf{u}_{2}=-\mathbb{L}(i \beta)^{-1} A\left(\partial_{x}\right) \mathbf{u}_{1}, \\
m_{2, p}=\frac{1}{i p \omega}\left(g\left(\mathbf{u}_{0}, \mathbf{u}_{1}\right)_{p}+g\left(\mathbf{u}_{1}, \mathbf{u}_{0}\right)_{p}, \quad p \neq 0 .\right.
\end{array}\right.
$$

\section{Terms of order $O(\sqrt{\varepsilon})$ :}

$$
\left\{\begin{array}{l}
L(p \beta) u_{3, p}+A\left(\partial_{x}\right) u_{2, p}+\partial_{t} u_{1, p}=f\left(\mathbf{u}_{0}, \mathbf{m}_{1}\right)_{p}+f\left(\mathbf{u}_{1}, \mathbf{m}_{0}\right) p \\
\omega \partial_{\theta} \mathbf{m}_{3}+\left(\partial_{t}+M\left(\partial_{x}\right)\right) \mathbf{m}_{1}=g\left(\mathbf{u}_{0}, \mathbf{u}_{2}\right)+g\left(\mathbf{u}_{2}, \mathbf{u}_{0}\right)+2 g\left(\mathbf{u}_{1}, \mathbf{u}_{1}\right) .
\end{array}\right.
$$

We set $\mathbf{u}_{3}=0, \mathbf{m}_{3}=0, P(p \beta) u_{2, p}=0$, and $m_{2,0}=0$. Then one has

$$
\begin{array}{r}
\left(\partial_{t}-i \omega^{\prime \prime}(p \beta)\left(\partial_{x}, \partial_{x}\right)\right) P(p \beta) u_{1, p}=P(p \beta) f\left(\mathbf{u}_{0}, \mathbf{m}_{1}\right)_{p}+f\left(\mathbf{u}_{1}, \mathbf{m}_{0}\right)_{p} \\
\left(\partial_{t}+M\left(\partial_{x}\right)\right) m_{1,0}=\left(g\left(\mathbf{u}_{0}, \mathbf{u}_{2}\right)+g\left(\mathbf{u}_{2}, \mathbf{u}_{0}\right)+2 g\left(\mathbf{u}_{1}, \mathbf{u}_{1}\right)\right)_{0}
\end{array}
$$


The weak transparency assumption implies that

$$
g\left(P(p \beta) \mathbf{u}_{1}, P(p \beta) \mathbf{u}_{1}\right)=0
$$

hence the system satisfied by $\left(P(p \beta) u_{1, p}, m_{1,0}\right)$ is linear. It depends on nonlinear, differential terms involving $\mathbf{U}_{0}$.

With the above equations, the profile defined by

$$
\mathbf{U}^{\varepsilon}:=\left(\mathbf{u}_{0}+\sqrt{\varepsilon} \mathbf{u}_{1}+\varepsilon(1-\mathbb{P}(\beta)) \mathbf{u}_{2}, m_{0,0}+\sqrt{\varepsilon} \mathbf{m}_{1}+\varepsilon \mathbf{m}_{2}\right),
$$

is an approximate solution to (3.41) at order 1 with an existence time $t^{*}>0$ independent of $\varepsilon$.

Remark 3.1 Note that the limit system (3.46)-(3.47) has the form (1.1). Without Assumption 1.5, we would have found

$$
\left\{\begin{array}{ccc}
i\left(\partial_{t}+c \partial_{z}\right) u+\Delta u & =n u \\
\partial_{t}^{2} n-\Delta n & =\Delta|u|^{2}
\end{array}\right.
$$

a system for which there exists no existence result if $c \neq 0$. Assumption 1.5 precisely ensures that $c=0$. A formal derivation of (3.51) can be found in [27].

\subsection{Example}

The limit system for (1.10) and (1.12) is

$$
\left\{\begin{array}{rr}
2 i \partial_{t} u+\Delta u= & -m u, \\
\partial_{t} m+\nabla \cdot m= & 0 \\
\partial_{t} m+\nabla m= & -2 \nabla|u|^{2},
\end{array}\right.
$$

where, with the notations of the previous section, $\omega=1, u=E_{0,1}$ and $m=$ $n_{0,0} \cdot$ 


\section{Stability of the approximate solution}

In this section, we establish our main result, that is the stability of the approximate solution constructed in section 3. As mentionned in the introduction, we are not in the weakly nonlinear regime of geometric optics, and classical results [8] do not imply the stability in our context.

We prove Theorem 1.8 which states an energy estimate in $H^{\sigma}$ norm for the linearized operator in the variables $t, x, \theta$. Its proof involves the following lemma (also used in [6]), whose proof is straightforward:

Lemma 4.1 For $\alpha>0$ and $u \in H^{\infty}\left(\mathbb{R}^{d} \times \mathbb{T}\right)$, one sets $\underline{u}:=\mathcal{F}^{-1}\left(\mathbf{1}_{\left\{|\eta| \leq \varepsilon^{-\alpha}\right\}} \mathcal{F} u\right)$, where $\mathbf{1}_{\left\{|\eta| \leq \varepsilon^{-\alpha}\right\}}$ is the characteristic function of $\left\{\eta,|\eta| \leq \varepsilon^{-\alpha}\right\} \subset \mathbb{R}^{d}$. $\underline{u}$ approximates $u$ in the following sense: for all $\sigma$,

$$
\|u-\underline{u}\|_{H^{\sigma}\left(\mathbb{R}^{d} \times \mathbb{T}\right)} \leq \sqrt{\varepsilon}\|u\|_{H^{\sigma^{\prime}}\left(\mathbb{R}^{d} \times \mathbb{T}\right)}
$$

with $\sigma^{\prime}=\sigma+\frac{1}{2 \alpha}$.

Then, with Proposition 1.14, Theorem 1.8 implies the nonlinear stability Theorem 1.9.

\subsection{Proof of Theorem 1.8}

One sets $F^{\varepsilon}:=\mathcal{L}_{1}^{\varepsilon}\left(U_{a}^{\varepsilon}, \partial_{t}, \partial_{x}, \beta \partial_{\theta} / \varepsilon\right) U^{\varepsilon}=\left(F_{1}^{\varepsilon}, F_{2}^{\varepsilon}\right) . t_{0}$ is fixed and $<t^{*}$. One defines the unitary groups of operators operating in $H^{\sigma}$, for all $\sigma$ :

$$
\begin{gathered}
S_{1}\left(\frac{t}{\varepsilon}\right):=\exp \left(\frac{t}{\varepsilon}\left(\frac{1}{\sqrt{\varepsilon}} A\left(\varepsilon \partial_{x}+k \partial_{\theta}\right)-\omega \partial_{\theta}+L_{0}\right)\right), \\
S_{2}\left(\frac{t}{\varepsilon}\right):=\exp \left(\frac{t}{\varepsilon}\left(B\left(\varepsilon \partial_{x}+k \partial_{\theta}\right)-\omega \partial_{\theta}\right)\right),
\end{gathered}
$$


and one performs the changes of variables (inpired by the "exponential cancellation" techniques of Schochet [23]):

$$
v(t):=S_{1}\left(\frac{t}{\varepsilon}\right) u(t), \quad n(t):=S_{2}\left(\frac{t}{\varepsilon}\right) m(t),
$$

to obtain

$$
\begin{aligned}
& \partial_{t} v(t)=S_{1}\left(\frac{t}{\varepsilon}\right) F_{1}^{\varepsilon} \\
& \partial_{t} n(t)=S_{2}\left(\frac{t}{\varepsilon}\right)\left(\frac{1}{\sqrt{\varepsilon}} g\left(u_{a}\right) S_{1}\left(-\frac{t}{\varepsilon}\right) v(t)+F_{2}^{\varepsilon}\right) .
\end{aligned}
$$

This gives

$$
\|v(t)\|_{H^{\sigma}} \leq\|v(0)\|_{H^{\sigma}}+\int_{0}^{t}\left\|F_{1}^{\varepsilon}\left(t^{\prime}\right)\right\|_{H^{\sigma}} d t^{\prime}
$$

and

$$
\left\|\partial_{t} v(t)\right\|_{H^{\sigma}} \leq\left\|F_{1}^{\varepsilon}(t)\right\|_{H^{\sigma}}
$$

It remains to obtain bounds independent of $\varepsilon$ for $n$. It suffices to bound the $L^{\infty}\left(\left[0, t_{0}\right], H^{\sigma}\left(\mathbb{R}^{d} \times \mathbb{T}\right)\right)$ norm of

$$
I^{\varepsilon}(t, x, \theta):=\frac{1}{\sqrt{\varepsilon}} \int_{0}^{t} S_{2}\left(\frac{t^{\prime}}{\varepsilon}\right) g\left(u_{a}\left(t^{\prime}, x, \theta\right)\right) S_{1}\left(-\frac{t^{\prime}}{\varepsilon}\right) v\left(t^{\prime}, x, \theta\right) d t^{\prime} .
$$

Indeed, if we obtain

$$
\left\|I^{\varepsilon}\right\|_{L^{\infty}\left(\left[0, t_{0}\right], H^{\sigma}\left(\mathbb{R}^{d} \times \mathbb{T}\right)\right)} \leq C\left(t_{0}\right)\left(\|v\|_{L^{\infty}\left(\left[0, t_{0}\right], H^{\sigma}\left(\mathbb{R}^{d} \times \mathbb{T}\right)\right)}+\left\|\partial_{t} v\right\|_{L^{\infty}\left(\left[0, t_{0}\right], H^{\sigma}\left(\mathbb{R}^{d} \times \mathbb{T}\right)\right)}\right)
$$

where $C\left(t_{0}\right)$ depends on $t_{0}$ but not on $\varepsilon$, then Gronwall's lemma allows one to conclude.

We now prove (4.52). In Fourier modes:

$$
\begin{aligned}
\hat{I}^{\varepsilon}(t, \eta, p)=\frac{1}{\sqrt{\varepsilon}} \int_{0}^{t} \sum_{p^{\prime}} \int & e^{i t^{\prime}(B(\varepsilon \eta+p k)-p \omega) / \varepsilon} g\left(\hat{u}_{a}\left(t^{\prime}, \eta-\eta^{\prime}, p-p^{\prime}\right)\right) \\
& \times e^{-i t^{\prime}\left(A\left(\varepsilon \eta^{\prime}+p^{\prime} k\right) / \sqrt{\varepsilon}-p^{\prime} \omega+L_{0}\right) / \varepsilon} \hat{v}\left(t^{\prime}, \eta^{\prime}, p^{\prime}\right) d t^{\prime} d \eta^{\prime} .
\end{aligned}
$$

The spectral decompositions read

$$
\frac{1}{\sqrt{\varepsilon}} A(i(\varepsilon \eta+p k))-i p \omega+L_{0}=: \sum_{s^{\prime}} i\left(-p \omega+\lambda_{s^{\prime}}^{\varepsilon}(\varepsilon \eta+p k)\right) P_{s^{\prime}}(\varepsilon \eta+p k)
$$




$$
B(i(\varepsilon \eta+p k))-i p \omega=: \sum_{s} i\left(-p \omega+\mu_{s}(\varepsilon \eta+p k)\right) Q_{s}(\varepsilon \eta+p k) .
$$

With the previous notation for the projectors, one has $P_{s^{\prime}}(\eta)=P\left(\lambda_{s^{\prime}}(\eta), \eta\right)$, and $Q_{s}(\eta)=Q\left(\mu_{s}(\eta), \eta\right)$. One sets

$$
\begin{aligned}
\varphi_{s, s^{\prime}}^{\varepsilon}\left(\varepsilon \eta, \varepsilon \eta^{\prime}, p, p^{\prime}\right) & :=\left(p^{\prime}-p\right) \omega+\mu_{s}(\varepsilon \eta+p k)-\lambda_{s^{\prime}}\left(\varepsilon \eta^{\prime}+p^{\prime} k\right), \\
\psi_{s, s^{\prime}}^{\varepsilon}\left(\varepsilon \eta^{\prime}, p, p^{\prime}\right) & :=\left(p^{\prime}-p\right) \omega+\mu_{s}\left(\varepsilon \eta^{\prime}+p k\right)-\lambda_{s^{\prime}}\left(\varepsilon \eta^{\prime}+p^{\prime} k\right), \\
\tilde{\mu}_{s}\left(\varepsilon \eta, \varepsilon \eta^{\prime}, p\right) & :=\varphi_{s, s^{\prime}}^{\varepsilon}\left(\varepsilon \eta, \varepsilon \eta^{\prime}, p, p^{\prime}\right)-\psi_{s, s^{\prime}}^{\varepsilon}\left(\varepsilon \eta^{\prime}, p, p^{\prime}\right) \\
& =\mu_{s}(\varepsilon \eta+p k)-\mu_{s}\left(\varepsilon \eta^{\prime}+p k\right) .
\end{aligned}
$$

$\hat{I}^{\varepsilon}$ decomposes as

$$
\hat{I}^{\varepsilon}(t, \eta, p)=\sum_{p^{\prime}} \sum_{s, s^{\prime}} \hat{I}_{p^{\prime}, s, s^{\prime}}^{\varepsilon}(t) .
$$

From now on, the indices $s, s^{\prime}$ are fixed and we suppress the dependances of the variables on these indices. We set

$$
\begin{gathered}
\xi:=\left(\mu_{s}(\varepsilon \eta+p k), \varepsilon \eta+p k\right), \quad \xi^{\prime}:=\left(p^{\prime} \omega+\lambda_{s^{\prime}}\left(\varepsilon \eta^{\prime}+p^{\prime} k\right), \varepsilon \eta^{\prime}+p^{\prime} k\right), \\
\xi^{\prime \prime}:=\left(\mu_{s}\left(\varepsilon \eta^{\prime}+p k\right), \varepsilon \eta^{\prime}+p k\right),
\end{gathered}
$$

and

$$
\hat{J}\left(u_{a}\right):=\hat{I}^{\varepsilon}(t, \eta, p)
$$

$\hat{J}\left(u_{a}\right)$ depends linearly on $u_{a}$. We want to find a uniform bound (with respect to $\varepsilon)$ for the $L^{\infty}\left(\left[0, t_{0}\right], H^{\sigma}\left(\mathbb{R}^{d} \times \mathbb{T}\right)\right)$ norm of $J\left(u_{a}\right)$, the inverse Fourier transform (with respect to $\eta$ and $p$ ) of $\hat{J}\left(u_{a}\right)$. We have

$$
\begin{aligned}
\hat{J}\left(u_{a}\right) & =\frac{1}{\sqrt{\varepsilon}} \int_{0}^{t} \sum_{p^{\prime}} \int e^{i t^{\prime} \varphi^{\varepsilon} / \varepsilon} Q(\xi) g\left(\hat{u}_{a}\left(t^{\prime}, \eta-\eta^{\prime}, p-p^{\prime}\right)\right) P\left(\xi^{\prime}\right) \hat{v}\left(t^{\prime}, \eta^{\prime}, p^{\prime}\right) d \eta^{\prime} d t^{\prime} \\
& =\frac{1}{\sqrt{\varepsilon}} \int_{0}^{t} \sum_{p^{\prime}} \int e^{i t^{\prime} \psi^{\varepsilon} / \varepsilon} Q(\xi) g\left(e^{i t^{\prime} \tilde{\mu} / \varepsilon} \hat{u}_{a}\left(t^{\prime}, \eta-\eta^{\prime}\right)\right) P\left(\xi^{\prime}\right) \hat{v}\left(t^{\prime}, \eta^{\prime}\right) d \eta^{\prime} d t^{\prime} .
\end{aligned}
$$

The end of the proof is organized as follows: A regularity argument (Lemma 4.1) for the approximate solution shows that the relevant phase is 
$\psi^{\varepsilon}$. Then for the frequencies $\eta^{\prime}$ such that $\psi^{\varepsilon}\left(\varepsilon \eta^{\prime}\right)$ is far from 0 , a non stationnary phase argument provide a uniform bound for the integral. When $\psi^{\varepsilon}$ is close to 0, Assumption 1.14 allows to obtain a uniform bound as well.

First consider $\hat{J}\left(u_{a}-\underline{u}_{a}\right)$, with the notation of Lemma 4.1. The parameter $\alpha$ will later be chosen small enough. In this integral, the rough majoration of the oscillating exponential by 1 gives

$$
\begin{aligned}
\left|\hat{J}\left(u_{a}-\underline{u}_{a}\right)\right| & \leq \frac{c}{\sqrt{\varepsilon}} \int_{0}^{t} \sum_{p^{\prime}} \int_{\eta^{\prime}}\left|\left(\hat{u}_{a}-\underline{\hat{u}}_{a}\right)\left(t^{\prime}, \eta-\eta^{\prime}, p-p^{\prime}\right)\right|\left|\hat{v}\left(t^{\prime}, \eta^{\prime}, p^{\prime}\right)\right| d \eta^{\prime} d t^{\prime} \\
& \leq \frac{c}{\sqrt{\varepsilon}} \int_{0}^{t}\left(\left|\left(\hat{u}_{a}-\underline{\hat{u}}_{a}\right)\right| *|\hat{v}|\right)\left(t^{\prime}, \eta, p\right) d t^{\prime},
\end{aligned}
$$

where $*$ denotes the convolution in the Fourier variables $\eta, p$. Using the notation $\left.<\eta, p>:=\left(1+|\eta|^{2}+p^{2}\right)^{1 / 2}\right)$ and Peetre's inequality

$$
<\eta, p>^{\sigma} \leq c_{\sigma}<\eta-\eta^{\prime}, p-p^{\prime}>^{\sigma}<\eta^{\prime}, p^{\prime}>^{\sigma},
$$

we have

$$
\begin{gathered}
\left\|J\left(u_{a}-\underline{u}_{a}\right)\right\|_{H^{\sigma}\left(\mathbb{R}^{d} \times \mathbb{T}\right)}^{2} \\
\leq \frac{C}{\varepsilon} \int_{0}^{t}\left\|\left(<\eta, p>^{\sigma / 2}\left|\hat{u}_{a}-\underline{\hat{u}}_{a}\right|\right) *\left(<\eta, p>^{\sigma / 2}|\hat{v}|\right)\right\|_{L^{2}\left(\mathbb{R}^{d} \times \mathbb{T}\right)}^{2} d t^{\prime} \\
\leq \frac{C}{\varepsilon} \int_{0}^{t}\left\|<\eta, p>^{\sigma / 2}\left|\hat{u}_{a}-\underline{\hat{u}}_{a}\right|\right\|_{L^{1}\left(\mathbb{R}^{d} \times \mathbb{T}\right)}^{2}\left\|<\eta, p>^{\sigma / 2}|\hat{v}|\right\|_{L^{2}\left(\mathbb{R}^{d} \times \mathbb{T}\right)}^{2} d t^{\prime} .
\end{gathered}
$$

Then using Hölder's inequality and Lemma 4.1,

$$
\begin{aligned}
\left\|<\eta, p>^{\sigma / 2}\left(\hat{u}_{a}-\underline{\hat{u}}_{a}\right)\right\|_{L^{1}\left(\mathbb{R}^{d} \times \mathbb{T}\right)} & \leq C(d)\left\|u_{a}-\underline{u}_{a}\right\|_{H^{\sigma^{\prime}\left(\mathbb{R}^{d} \times \mathbb{T}\right)}} \\
& \leq C(d) \sqrt{\varepsilon}\left\|u_{a}\right\|_{H^{\sigma^{\prime \prime}\left(\mathbb{R}^{d} \times \mathbb{T}\right)},}
\end{aligned}
$$

where $C(d)$ depends only on $d, \sigma^{\prime}=\sigma+d / 2$ and $\sigma^{\prime \prime}=\sigma^{\prime}+\frac{1}{2 \alpha}$. This yields $\left\|J\left(u_{a}-\underline{u}_{a}\right)\right\|_{L^{\infty}\left(\left[0, t_{0}\right], H^{\sigma}\left(\mathbb{R}^{d} \times \mathbb{T}\right)\right)} \leq C\left(t_{0}\right)\left\|u_{a}\right\|_{L^{\infty}\left(\left[0, t_{0}\right], H^{\sigma^{\prime \prime}}\left(\mathbb{R}^{d} \times \mathbb{T}\right)\right)}\|v\|_{L^{\infty}\left(\left[0, t_{0}\right], H^{\sigma}\left(\mathbb{R}^{d} \times \mathbb{T}\right)\right)}$.

Consider now $\hat{J}\left(\underline{u}_{a}\right)$. One sets $D^{\varepsilon}:=\left\{\eta^{\prime} \in \mathbb{R}^{d},\left|\psi^{\varepsilon}\left(\varepsilon \eta^{\prime}\right)\right| \leq \sqrt{\varepsilon}\right\}$. The integral over $D^{\varepsilon}$ is denoted by $\hat{J}_{2}$ and the integral over the complement of $D^{\varepsilon}$ in $\mathbb{R}^{d}$ is denoted by $\hat{J}_{1}$. 
Integrating by parts, one has $\hat{J}_{1}=\hat{J}_{1,1}+\hat{J}_{1,2}$, with

$$
\begin{aligned}
\hat{J}_{1,1}= & \frac{1}{\sqrt{\varepsilon}} \sum_{p^{\prime}} \int_{\mathbb{R}^{d} \backslash D^{\varepsilon}}\left(i \frac{\psi^{\varepsilon}}{\varepsilon}\right)^{-1} Q g\left(e^{i t \tilde{\mu} / \varepsilon} \hat{u}_{a}\left(t, \eta-\eta^{\prime}, p-p^{\prime}\right)\right) P \hat{v}\left(t, \eta^{\prime}, p^{\prime}\right) d \eta^{\prime} \\
& -\frac{1}{\sqrt{\varepsilon}} \sum_{p^{\prime}} \int_{\mathbb{R}^{d} \backslash D^{\varepsilon}}\left(i \frac{\psi^{\varepsilon}}{\varepsilon}\right)^{-1} Q g\left(\underline{\hat{u}}_{a}\left(0, \eta-\eta^{\prime}, p-p^{\prime}\right)\right) P \hat{v}\left(0, \eta^{\prime}, p^{\prime}\right) d \eta^{\prime}
\end{aligned}
$$

and

$\hat{J}_{1,2}=\frac{1}{\sqrt{\varepsilon}} \int_{0}^{t} \sum_{p^{\prime}} \int_{\mathbb{R}^{d} \backslash D^{\varepsilon}}\left(i \frac{\psi^{\varepsilon}}{\varepsilon}\right)^{-1} Q \partial_{t}\left(g\left(e^{i t^{\prime} \tilde{\mu} / \varepsilon} \underline{\underline{u}}_{a}\left(t^{\prime}, \eta-\eta^{\prime}, p-p^{\prime}\right)\right) P \hat{v}\left(t^{\prime}, \eta^{\prime}, p^{\prime}\right)\right) d \eta^{\prime} d t^{\prime}$.

$\hat{J}_{1,1}$ can be directly bounded as

$$
\left|\hat{J}_{1,1}\right| \leq C\left(\left|\underline{\hat{u}}_{a}\right| *|\hat{v}|(t)-\left|\underline{\hat{u}}_{a}\right| *|\hat{v}|(0)\right) .
$$

This gives again

$\left\|J_{1,1}\right\|_{L^{\infty}\left(\left[0, t_{0}\right], H^{\sigma}\right)} \leq C\left(t_{0}\right)\left(\left\|u_{a}\right\|_{L^{\infty}\left(\left[0, t_{0}\right], H^{\sigma^{\prime \prime}}\right)}\|v\|_{L^{\infty}\left(\left[0, t_{0}\right], H^{\sigma}\right)}+\left\|u_{a}(0)\right\|_{H^{\sigma^{\prime \prime}}}\|v(0)\|_{H^{\sigma}}\right)$.

One has

$$
\frac{1}{\varepsilon} \tilde{\mu}=\frac{1}{\varepsilon}\left(\mu_{s}(\varepsilon \eta+p k)-\mu_{s}\left(\varepsilon \eta^{\prime}+p k\right)\right) \stackrel{\varepsilon \rightarrow 0}{\rightarrow} \mu_{s}^{\prime}(p k)\left(\eta^{\prime}-\eta\right),
$$

where $\mu_{s}^{\prime}(p k) \eta_{0}$ is the derivative of $\mu_{s}$ at $p k$ in the direction $\eta_{0}$. As $\mu_{s}$ is homogeneous of degree 1 (see the definition of Char $M$ ), $\mu_{s}^{\prime}$ is homogeneous and degree 0 , in particular, it is bounded. Thus there exists $\underline{C}$ such that for all $s$, one has $\tilde{\mu} \leq \underline{\varepsilon} C\left|\eta-\eta^{\prime}\right|$. One has

$$
\begin{gathered}
\hat{J}_{1,2}=\frac{1}{\sqrt{\varepsilon}} \int_{0}^{t} \sum_{p^{\prime}} \int_{\mathbb{R}^{d} \backslash D^{\varepsilon}}\left(i \frac{\psi^{\varepsilon}}{\varepsilon}\right)^{-1} e^{i t \psi^{\varepsilon} / \varepsilon} \\
Q g\left(e^{i t^{\prime} \tilde{\mu} / \varepsilon}\left(\frac{i \tilde{\mu}}{\varepsilon} \underline{u}_{a}+\partial_{t} \hat{u}_{a}\right)\left(t^{\prime}, \eta-\eta^{\prime}, p-p^{\prime}\right)\right) P \hat{v}\left(t^{\prime}, \eta^{\prime}, p^{\prime}\right) d \eta^{\prime} d t^{\prime} \\
+\frac{1}{\sqrt{\varepsilon}} \int_{0}^{t} \sum_{p^{\prime}} \int_{\mathbb{R}^{d} \backslash D^{\varepsilon}}\left(i \frac{\psi^{\varepsilon}}{\varepsilon}\right)^{-1} e^{i t \psi^{\varepsilon} / \varepsilon} \\
Q g\left(e^{i t^{\prime} \tilde{\mu} / \varepsilon} \underline{\hat{u}}_{a}\left(t^{\prime}, \eta-\eta^{\prime}, p-p^{\prime}\right)\right) P \partial_{t} \hat{v}\left(t^{\prime}, \eta^{\prime}, p^{\prime}\right) d \eta^{\prime} d t^{\prime} .
\end{gathered}
$$

Hence

$$
\left|\hat{J}_{1,2}\right| \leq C\left(t_{0}\right) \int_{0}^{t}\left(\left(|\eta|\left|\underline{\hat{u}}_{a}+\right| \partial_{t} \underline{\hat{u}}_{a} \mid\right) *|\hat{v}|+\left|\underline{\hat{u}}_{a}\right| *\left|\partial_{t} \hat{v}\right|\right) d t^{\prime}
$$


and this yields as above

$$
\left\|J_{1,2}\right\|_{L^{\infty}\left(\left[0, t_{0}\right], H^{\sigma}\right)} \leq C\left(t_{0}\right)\left(\|v\|_{L^{\infty}\left(\left[0, t_{0}\right], H^{\sigma}\left(\mathbb{R}^{d} \times \mathbb{T}\right)\right)}+\left\|\partial_{t} v\right\|_{L^{\infty}\left(\left[0, t_{0}\right], H^{\sigma}\left(\mathbb{R}^{d} \times \mathbb{T}\right)\right)}\right)
$$

where $C\left(t_{0}\right)$ depends on $\left\|u_{a}\right\|_{L^{\infty}\left(\left[0, t_{0}\right], H^{\sigma^{\prime \prime}+1}\right)}$ and $\left\|\partial_{t} u_{a}\right\|_{L^{\infty}\left(\left[0, t_{0}\right], H^{\sigma}\right)}$.

It remains to bound $\hat{J}_{2}$. The interaction coefficient that appears in $\hat{J}_{2}$ is

$$
Q(\xi) g\left(\underline{\hat{u}}_{a}\left(\eta-\eta^{\prime}, p-p^{\prime}\right)\right) P\left(\xi^{\prime}\right) \hat{v}\left(\eta^{\prime}, p^{\prime}\right) .
$$

By linearity of $g$, we can bound separately the contribution of

$$
P\left(\left(p-p^{\prime}\right) \beta\right) \underline{\hat{u}}_{a}\left(\eta-\eta^{\prime}, p-p^{\prime}\right)
$$

and the contribution of

$$
\left(1-P\left(\left(p-p^{\prime}\right) \beta\right)\right) \underline{\hat{u}}_{a}\left(\eta-\eta^{\prime}, p-p^{\prime}\right) .
$$

By assumption on $u_{a}$

$$
\left(1-P\left(\left(p-p^{\prime}\right) \beta\right)\right) \underline{\hat{u}}_{a}\left(\eta-\eta^{\prime}, p-p^{\prime}\right)=O(\sqrt{\varepsilon}),
$$

hence the contribution of this term is bounded as above. It remains to bound the contribution of

$$
Q(\xi) g\left(P\left(\left(p-p^{\prime}\right) \beta\right) \underline{\hat{u}}_{a}\left(\eta-\eta^{\prime}, p-p^{\prime}\right)\right) P\left(\xi^{\prime}\right) \hat{v}\left(\eta^{\prime}, p^{\prime}\right) \text {. }
$$

We have

$$
\left|\xi^{\prime \prime}-\xi\right|=\left|\left(\tilde{\mu}_{s}\left(\varepsilon \eta, \varepsilon \eta^{\prime}, p\right), \varepsilon\left(\eta-\eta^{\prime}\right)\right)\right|=O\left(\varepsilon\left|\eta-\eta^{\prime}\right|\right) \leq C \varepsilon^{1-\alpha}
$$

By analyticity of $Q$ (which is guarantied by the axisymmetry hypothesis on Char $M$ ), we thus have

$$
Q\left(\xi^{\prime \prime}\right)-Q(\xi)=O\left(\varepsilon^{1-\alpha}\right)
$$

and choosing $0<\alpha<1 / 2$, we can change $Q(\xi)$ into $Q\left(\xi^{\prime \prime}\right)$ in (4.54). For $\eta^{\prime} \in D^{\varepsilon}$, one has $\left|\psi^{\varepsilon}\right| \leq \sqrt{\varepsilon}$. This is the hypothesis of Assumption 1.7. Set 
$D_{1}^{\varepsilon}:=\left\{\eta^{\prime} \in D^{\varepsilon},\left|\psi^{\varepsilon}\left(\varepsilon \eta^{\prime}\right)\right| \leq \varepsilon\right\}$. The integral over $D_{1}^{\varepsilon}$ is denoted by $\hat{J}_{2,1}$ and the integral over the complement of $D_{1}^{\varepsilon}$ in $D^{\varepsilon}$ is denoted by $\hat{J}_{2,2}$. For $\eta^{\prime} \in D_{1}^{\varepsilon}$, using Assumption 1.7, we have the bound $\left|Q\left(\xi^{\prime \prime}\right) g\left(P\left(\left(p-p^{\prime}\right) \beta\right) \underline{\hat{u}}_{a}\left(\eta-\eta^{\prime}, p-p^{\prime}\right)\right) P\left(\xi^{\prime}\right) \hat{v}\left(\eta^{\prime}, p^{\prime}\right)\right| \leq C \sqrt{\varepsilon}\left|\underline{\hat{u}}_{a}\left(\eta-\eta^{\prime}, p-p^{\prime}\right)\right|\left|\hat{v}\left(\eta^{\prime}, p^{\prime}\right)\right|$.

This gives as above

$$
\left|\hat{J}_{2,1}\right| \leq C \int_{0}^{t} \sum_{p^{\prime}} \int_{\eta^{\prime}}\left|\underline{\hat{u}}_{a}\left(t^{\prime}, \eta-\eta^{\prime}, p-p^{\prime}\right)\right|\left|\hat{v}\left(t^{\prime}, \eta^{\prime}, p^{\prime}\right)\right| d \eta^{\prime} d t^{\prime} .
$$

Finally, for $\eta^{\prime}$ in $D^{\varepsilon} \backslash D_{1}^{\varepsilon}$, that is such that $\varepsilon \leq\left|\psi^{\varepsilon}\left(\varepsilon \eta^{\prime}\right)\right| \leq \sqrt{\varepsilon}$, Assumption 1.7 implies

$\frac{\varepsilon}{\left|\psi^{\varepsilon}\left(\varepsilon \eta^{\prime}\right)\right|}\left|Q\left(\xi^{\prime \prime}\right) g\left(P\left(\left(p-p^{\prime}\right) \beta\right) \underline{\hat{u}}_{a}\left(\eta-\eta^{\prime}, p-p^{\prime}\right)\right) P\left(\xi^{\prime}\right) \hat{v}\left(\eta^{\prime}, p^{\prime}\right)\right| \leq C \sqrt{\varepsilon}\left|\underline{\hat{u}}_{a}\left(\eta-\eta^{\prime}\right)\right|\left|\hat{v}\left(\eta^{\prime}\right)\right|$.

An integration by parts then gives a bound for $J_{2,2}$ of the same type as the bound for $J_{1,2}$. Gathering the pieces of the majoration of $J$ together, one finds the desired bound for $n$.

\section{References}

[1] B. Bidégaray. On a nonlocal Zakharov equation. Nonlinear Analysis 1996, $25(3), 247-278$.

[2] C. Cheverry, O. Guès, G. Métivier. Oscillations fortes sur un champ linéairement dégénéré. Ann. Sci. cole Norm. Sup. (4) 2003, 36 (5), $691-745$.

[3] M. Colin, T. Colin. On a quasilinear Zakharov system arising in plasma physics. Differential and Integral Equations 2004, 17 (3-4), 297-330.

[4] T. Colin. Rigorous derivation of the nonlinear Schrdinger equation and Davey-Stewartson systems from quadratic hyperbolic systems. Asymptotic Analysis 2002, 31 (1), 69-91. 
[5] T. Colin, C. Galusinski, H. Kaper. Waves in ferromagnetic media. Comm. Part. Diff. Eq. 2002, 27 (7-8), 1625-1658.

[6] T. Colin, D. Lannes. Long wave-short wave resonance for nonlinear geometric optics. Duke Math. Journal 2001, 107 (2), 351-419.

[7] J-L. Delcroix and A. Bers. Physique des plasmas 1, 2; Inter EditionsEditions du CNRS: Paris, 1994.

[8] J.-L. Joly, G. Métivier, J. Rauch. Diffractive nonlinear geometric optics with rectification. Indiana Univ. Math. Journal 1998, 47, 1167-1241.

[9] P. Donnat, J.-L. Joly, G. Métivier, J. Rauch. Dispersive nonlinear geometric optics. Séminaire Equations aux Dérivées partielles de l' Ecole Polytechnique 1995-1996, XVII, 1-23.

[10] J.-L. Joly, G. Métivier, J. Rauch. Transparent nonlinear geometric optics and Maxwell-Bloch equations. Journal of Differential Equations 2000, 166, 175-250.

[11] J. Ginibre, Y. Tsutsumi and G. Velo. On the Cauchy problem for the Zakharov system. J. Funct. Anal. 1997, 151, 384-436.

[12] L. Glangetas and F. Merle. Existence of self-similar blow-up solutions for the Zakharov equation in dimension two I. Comm. Math. Phys. 1994, 160 (1), 173-215.

[13] L. Glangetas and F. Merle. Concentration properties of blow up solutions and instability results for Zakharov equation in dimension two II, Comm. Math. Phys., 1994, 160 (2), 349-389.

[14] R. Glassey. Convergence of an energy-preserving scheme for the Zakharov equations in one space dimension. Math. Comp. 1992, 197, $83-102$. 
[15] R. Glassey, Approximate solutions to the Zakharov equations via finite differences. J. Comput. Phys. 1992, 100 (2), 377-383.

[16] T. Kato, Perturbation theory for linear operators 2nd Ed; Grundlehren der Mathematischen Wissenschaften 132; Springer Verlag: Berlin-New York, 1966.

[17] D. Lannes. Dispersive effects for nonlinear diffractive geometrical optics with rectification. Asymptotic Analysis 1998, 18, 111-146.

[18] A. Majda, Compressible fluid flows and systems of conservation laws in several space variables; Applied Mathematical Sciences 53; Springer Verlag: New York, 1984.

[19] T.Ozawa, K. Tsutaya, Y. Tsutsumi. Global existence and asymptotic behavior of solutions for the Klein-Gordon equations with quadratic nonlinearity in two space dimensions. Math. Z. 1996, 222 (3), 341362.

[20] T. Ozawa and Y. Tsutsumi. Existence and smoothing effect of solution for the Zakharov equations. Publ. Res. Inst. Math. Sci 1992, 28 (3), 329-361.

[21] G. Riazuelo. Etude théorique et numérique de l'influence du lissage optique sur la filamentation des faisceaux lasers dans les plasmas souscritiques de fusion inertielle; Université Paris XI, 2001.

[22] D.A. Russel, D.F. Dubois and H.A. Rose. Nonlinear saturation of simulated Raman scattering in laser hot spots. Physics of Plasmas 1999, $6(4), 1294-1317$.

[23] S. Schochet. Fast singular limits of hyperbolic PDEs, Journal of Differential Equations 1994, 114 (2), 476-512. 
[24] S. Schochet and M. Weinstein. The nonlinear Schrödinger limit of the Zakharov equations governing Langmuir turbulence. Comm. Math. Phys. 1986, 106, 569-580.

[25] C. Sulem and P-L. Sulem. The nonlinear Schrödinger Equation. SelfFocusing and Wave Collapse; Applied Mathematical Sciences 139; Springer, 1999.

[26] B. Texier. The short wave limit for nonlinear, symmetric hyperbolic systems. Adv. Diff. Eq. 2004, 8 (1), 1-51.

[27] B. Texier. WKB asymptotics for the Euler-Maxwell system, submitted.

[28] V.E. Zakharov, S.L. Musher and A.M. Rubenchik. Hamiltonian approach to the description of nonlinear plasma phenomena. Phys. Reports 1985, 129, 285-366. 